\title{
Earthquake Surface Slip-Length Data is Fit by Constant Stress Drop and is Useful for Seismic Hazard Analysis
}

\author{
by Bruce E. Shaw
}

\begin{abstract}
We present a new method to use directly observable surface-slip measurements in seismic hazard estimates. We present measures of scaling-relation fits to sliplength data. These fits show sublinear scaling, a slowing in the rate of slip increase for the longest ruptures, so that $L$ scaling — scaling with the length of the rupture-does not hold out to very large aspect ratio events. We find the best fitting for a constant stressdrop model, followed next by a square root of length model. The constant stress-drop model, newly introduced here, provides a geometrical explanation for a long-standing puzzle of why slip only begins to saturate at large aspect ratios. The good fit of the constant stress-drop model to the slip-length data lends further support to the observations of constant stress-drop scaling across the whole range of magnitudes of earthquakes, from small to great earthquakes. The good fit of the constant stress-drop model is also reflected by the low variability about the mean, with an average of less than a factor-of- 2 variability in stress drop about the mean observed. Converting magnitude-area scaling into implied slip-length scaling, we determine qualitative consistency in the functional forms, but a quantitative difference of, on average, 30\% more slip estimated from magnitude area compared with slip length.
\end{abstract}

Online Material: Tables of magnitude-length-width, magnitude-area, and surface slip-length relations.

\section{Introduction}

For more than a quarter of a century since Scholz (1982) pointed out the surprising observation that the slip of large earthquakes continued to increase for lengths far beyond the seismogenic depth, scientists have sought an explanation for this puzzling observation. A wide range of possibilities have been proposed, including the theories that slip might be penetrating deep below the seismogenic zone during very large ruptures (Das, 1982; King and Wesnousky, 2007; Hillers and Wesnousky, 2008; Shaw and Wesnousky, 2008); that multiple faults length scales may be playing a role (Manighetti et al., 2007); that large slips in the initiation of ruptures are leading to larger-scale ruptures (Bodin and Brune, 1996); and that large earthquakes are somehow different than small ones, with a breakdown of the constant stress-drop scaling observed in small earthquakes (Hanks, 1977) implied in a variety of proposed empirical relations (Wells and Coppersmith, 1994; Working Group on California Earthquake Probabilities, WGCEP, 2003 [WGCEP03]; Hanks and Bakun, 2008). These questions have important implications for the physics of earthquakes and for society, since scaling relations play a central role in current seismic hazard estimates (WGCEP, 2008). Current seismic hazard estimates have favored empirical scaling relations which infer a breakdown in constant stress-drop scaling, with increasing stress drops for the largest earthquakes (Wells and Coppersmith, 1994; WGCEP, 2003; Hanks and Bakun, 2008). However, this has led to a conundrum, with inconsistencies arising in attempts to reproduce empirical ground-motion relations using parameterized kinematic ruptures (Graves et al., 2011). Clearly, a better understanding is needed.

Here, we show that a very simple explanation, based on the geometrical implications of the transition from small, circular ruptures to large, long, rectangular ruptures offers a scaling relation that (1) matches well observed data of surface slip versus rupture length, (2) gives a large crossover length scale matching that observed for magnitude versus length observations (Romanowicz, 1992, 1994), and (3) gives constant stress-drop scaling for large earthquakes, which matches with that observed for small earthquakes, as well. We thus find a simple explanation for very important observations which have flummoxed scientists for decades.

Next, we demonstrate how surface-slip observations can be usefully incorporated into seismic hazard analysis. (This is a somewhat involved topic, so readers just interested in the constant stress-drop scaling relationship, and not the implications for seismic hazard, may turn now to the section 
Constant Stress Drop in Surface Slip-Length Scaling and skip the rest.) Seismic hazard estimates use a series of empirical relations to estimate shaking hazard. One set of geologically observable measurements, in which slip at the surface scales with rupture length, has not been traditionally used in these hazard estimates. In this paper, we show how seismic hazard estimates can usefully incorporate these measurements.

Seismic hazard analysis, as currently practiced, uses a number of empirical relations for various rupture and fault-based parameters to derive expected rates of magnitudes of events, and from that, shaking hazards associated with the events. To estimate rates of magnitudes of events, both the sizes and rates of events need to be estimated. To estimate sizes of events, empirical relations giving magnitude as a function of the log of the rupture area have been found to be quite useful across the broad range of scales relevant to seismic hazard.

Magnitude-area scaling has also been used to help determine the rates of events. It is this method that we argue in this paper can be improved by also considering surface slip-length scaling measurements. To define rates of events, a distribution of event sizes, which include the relative rates, is constructed. Despite its difficulty and uncertainty, it is a necessary step. The uncertainty is dealt with through different branches in a logic tree, reflecting various underlying distributions. Then, given a distribution of event sizes, rates of events are determined by placing various forms of integral constraints on the distributions. A common one examines the summed seismic moment and determines an overall rate of events by matching the average seismic moment rate to that of a target. For locations where faults are relatively well known, this constraint has been used through the technique of moment balancing. Here, slip rates on faults are combined with an estimate of downdip fault width to yield a long-term moment-release rate. While this approach works and allows for consistent budgeting, two major sources of uncertainty have been identified by using it. One source of uncertainty concerns the magnitude-area relations used to perform the moment balancing, with differences between the different magnitude-area relations believed to be one of the dominant uncertainties in some recent state-of-the-art hazard estimates (WGCEP, 2008). A second source of uncertainty concerns the downdip width of ruptures and how the moment is distributed with depth. Seismogenic depths have been determined using the hypocenters of small events. However, it is unclear how efficiently the origin points of these events document the depths to which ruptures propagate and release significant slip during large events.

In this paper, we propose a new alternative pathway for imposing integral constraints, one that uses observed surface slips as a basis to balance slip rates rather than moment rates. This alternative approach has a number of advantages. First, it manages to avoid some of the uncertainty surrounding the issue of the depth of rupture in large events. Second, it adds rate-calculation methods that do not depend on modulus estimates and their corresponding uncertainties. Third, it intro- duces into the analysis a new, independent, geologically observable dataset. Finally, by using the linear measure of slip rather than the logarithmic measure of magnitude as the independent variable, fits to the scaling relations are more naturally mapped onto the linearly summed integral constraints. Thus, errors are better accounted for where they matter most.

The scaling relation we examine in this paper for use in slip-rate balancing is average surface slip as a function of surface-rupture length. We examine fits of scaling relations to the slip-length data and determine that uncertainties compare favorably with magnitude-area uncertainties. We also determine that the best fits to the data show a saturation of slip at the largest length scales. The new constant stressdrop scaling is shown to give the best fit to the data, followed closely by a square root of length scaling. Finally, we compare similarities and differences between slip-length and magnitude-area scaling, and suggest how these can be applied to seismic hazard estimates.

The next section presents the new constant stress-drop slip-length scaling and demonstrates how accurately it fits the data. The paper then turns to an examination of how to apply slip-length scaling more generally to seismic hazard analysis. The section Magnitude-Area Scaling and Implied Slip-Length Scaling contains a discussion of the current basis for seismic hazard analysis, magnitude-area scaling relations, and how analogous implied slip-length scaling relations can be derived from them. We explore how accurately the implied slip-length scaling relations fit the data. In the section Surface Slip, we compare various slip-length scaling relations with the surface-slip data, and compare against implied slip-length scaling from magnitude-area data. The section, Incorporating Results into Hazard discusses applying this newslip-length pathway to seismic hazard analysis. Finally, the paper concludes.

\section{Constant Stress Drop in Surface Slip-Length Scaling}

We derive a new constant stress-drop slip-length scaling relation by considering how slip scales with length for ruptures breaking the surface of the Earth. For ruptures barely breaking the surface, we consider the slip of a circular rupture and examine the average slip as a function of length for a line intersecting the circle, ignoring free-surface effects at this stage. For a circular rupture with constant stress drop $\Delta \sigma$ on the interior circular rupture of radius $R$, of shear modulus $\mu$, slip is given by $S(r)=\frac{\Delta \sigma}{\mu} \frac{24}{7 \pi} \sqrt{R^{2}-r^{2}}$ where $S(r)$ is the slip across the fault as a function of the distance $r$ from the center of the circle. For a line of length $L$ intersecting this circle, the average slip $S$ on the line is $S=\frac{1}{L / 2} \int_{0}^{L / 2} \frac{\Delta \sigma}{\mu} \frac{24}{7 \pi} \sqrt{R^{2}-\left(x^{2}+y^{2}\right)} d x$ where $x$ is the distance along the line, and $y$ is the closest distance of the line from the center of the circle. A little algebra gives

$$
S=\frac{\Delta \sigma}{\mu} \frac{3}{7} L
$$


This is a new result, and is especially interesting in that it is independent of the radius of the circle $R$ and similarly the closest distance of the line from the center of the circle $y$, depending only on the length $L$ of the intersection.

The scaling for the limit of very long ruptures, ruptures much greater than the width $W$ of the rupture, is derived from the solution for infinite long ruptures of width $2 W$, with the factor of 2 now accounting for the free-surface effect. Average slip for these long rectangular ruptures is well known (e.g., Scholz, 2002), and is given by

$$
S=\frac{\Delta \sigma}{\mu} 2 W
$$

for strike-slip events. Treating the stiffnesses in parallel, we can combine these two limits into one scaling relation across the range of scales as

$$
S=\frac{\Delta \sigma}{\mu} \frac{1}{\frac{7}{3 L}+\frac{1}{2 W}},
$$

which gives our constant stress drop-scaling relation for strike-slip faults.

Generalizing this to allow for the possibility that stress drops might vary as a function of length, we can write this as

$$
S=\frac{1}{\frac{7}{3 L \Delta \sigma_{0} / \mu}+\frac{1}{2 W \Delta \sigma_{\infty} / \mu},}
$$

where $\Delta \sigma_{0}$ is the stress drop at smaller lengths and $\Delta \sigma_{\infty}$ is the stress drop at larger lengths. We can use this generalized form to test for constant stress drop in the data.

How does this compare with the data? Figure 1 shows surface slip-length data for large events, adapted from a dataset compiled by Wesnousky (2008). That paper also discusses sources of uncertainties in the data, a topic to which we will later return when we begin comparing fits of various scaling relations. The data combines all the various focal mechanisms, including strike-slip events shown with circles, normal events with diamonds, and thrust events with squares. We have modified the value for the 1857 M 7.8 Fort Tejon earthquake to reflect the recent light detection and ranging (LiDAR) results of (Zielke et al., 2010) regarding the overestimate of the original (Sieh, 1978) analysis for the Carizzo Plain section of that rupture; this correction leads to a $10 \%$ reduction in the average slip for that event. We have also added the value for the 2008 M 7.9 Wenchuan earthquake, using data from (Xu et al., 2009), shown with error bars given their estimate of 3-4 m of average slip at the surface; this provides a rare, extended rupture-length dip-slip event. For now, we will focus on the strike-slip events, since that is where we have the most data; later, we will discuss how our results generalize to the dip-slip events. These data are reproduced in (E) Table S3 in the electronic supplement to this paper.

The dashed lines in Figure 1 show the two asymptotic scalings, one for the smaller length $L$ scaling limit, equation (1), and one for the larger length $W$ scaling limit, equation (2). The solid line shows the combined scaling in

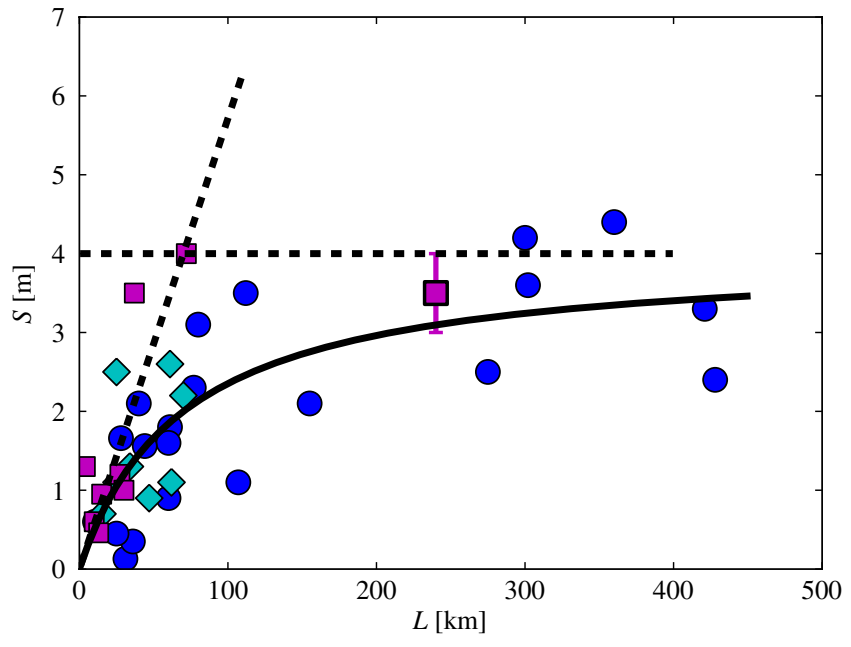

Figure 1. Geological surface slip observations of average slip versus length. Symbol types indicate focal mechanisms: strike-slip (circle), normal (diamond), and thrust (square). Dashed lines show asymptotic scaling limits for circular and long rectangular ruptures. Solid line shows scaling combining these two limits, equation (3). Parameters on lines are: constant stress drop $\Delta \sigma=4 \mathrm{MPa}$, and seismogenic width $W=15 \mathrm{~km}$. The color version of this figure is available only in the electronic edition.

parallel of these two stiffnesses, given in equation (3). Here, we assume a physical length scale for $W$, with $W=15 \mathrm{~km}$ for vertical strike-slip events. We also plot, for illustration, a stress-drop value of $\Delta \sigma=4 \mathrm{MPa}$, using a modulus $\mu=30 \mathrm{GPa}$, for all the curves. We immediately make an interesting observation: without optimizing, we see a very good fit for these quite physically reasonable values.

By combining the two scalings, assuming constant stress drop, we discover another interesting result: an explanation for the large crossover length scale from $L$ scaling to $W$ scaling. Here, the crossover scale comes from the quite different coefficients on the circular versus rectangular rupture, with a crossover length $L_{c}$ in the scaling at lengths

$$
L_{c}=\frac{14}{3} W \text {. }
$$

This large multiple of $W$ for the crossover value of $L_{c}$ provides an explanation for large-length scale crossovers found empirically by Romanowicz $(1992,1994)$ in moment-length scaling, and by others in parameterizations of the surface slip-length data (Shaw and Scholz, 2001; Manighetti et al., 2007; Shaw and Wesnousky, 2008). Taking $W=15 \mathrm{~km}$ gives a crossover length scale of $70 \mathrm{~km}$, which is very similar to the crossover length scale found seismologically (Romanowicz, 1992, 1994). The coefficient $14 / 3$ also explains much of the trend in the $S / W$ versus moment plot based on source inversions of Mai and Beroza (2000).

\section{Fitting Data}

To test the constant stress-drop hypothesis, we turn to equation (4). Here, we see that there is a degree of ambiguity in that there is a trade-off between changing values of $W$ and 


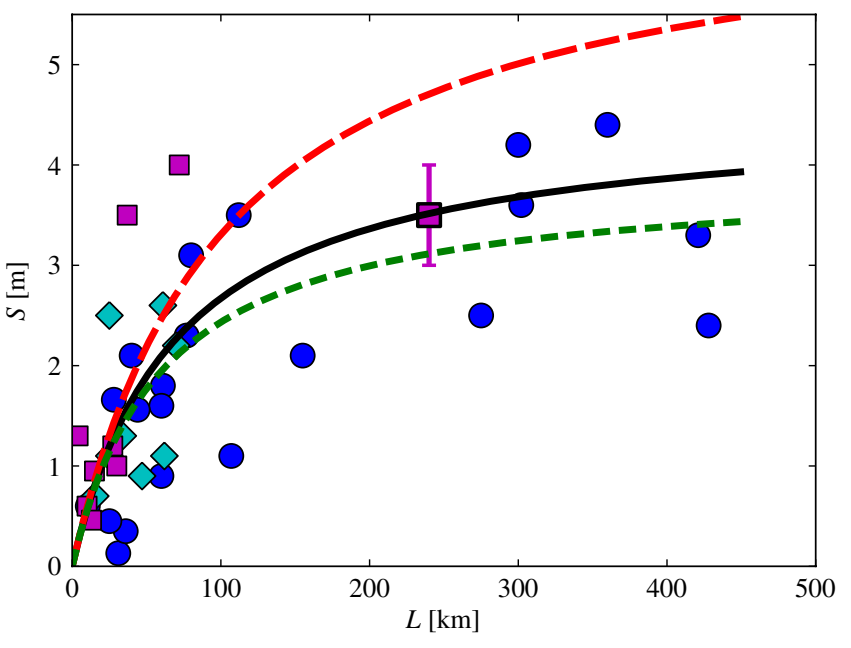

Figure 2. Dip effects on expected scaling. Data points are geological surface-slip observations of average slip versus length. Symbol types indicate focal mechanisms: strike-slip (circle), normal (diamond), and thrust (square). Curves show scaling expected for fixed seismogenic depth, but changing dip, and thus downdip width and changing modulus effects. All curves have the same stress drop, a value for best fit stress drop to strike-slip data of 3.91 MPa. Different curves use the same seismogenic depth of $15 \mathrm{~km}$ and different dips $90^{\circ}$ (solid line) strike-slip equation (3), and $60^{\circ}$ (short-dashed line) and $30^{\circ}$ (long-dashed line) dip-slip equation (7). Note only relatively small differences are expected in the scaling of events with different focal mechanisms, which appears consistent with data. The color version of this figure is available only in the electronic edition.

$\Delta \sigma_{\infty}$. One way to deal with this is to fix $W$ by assuming a physical origin for it. Taking $W=15 \mathrm{~km}$, a typical seismogenic depth that would then represent the downdip width $W$ on vertical strike-slip faults, we can fit the data in Figure 1, allowing for varying stress drops. Fixing $W$ to be a seismogenic width, and allowing for one stress-drop parameter on the $L$ scaling regime in addition to a second stress-drop parameter on the $W$ scaling regime as in equation (4), we can fit the two-parameter model and determine how much the two stress drops differ. Using only the strike-slip data, the difference in the stress drops is only $30 \%$. The Akaike information criteria (AIC; 50.05 and 51.55 for the one- and two-parameter models, respectively) and Fisher $F$-tests $[F(1,19)=0.460 p \approx 0.5]$ of the differences in misfit show the small additional gain in fitting for the two stress-drop parameter model, equation (4), relative to the one stress-drop parameter model, equation (3), is not justified by the cost of an additional parameter. (For more information on these types of statistical tests, see the caption for Table A1 in Appendix A, including additional related discussion within the manuscript.) Thus, this slight difference in best-fit values supports the constant stress-drop hypothesis, equation (3), as does the information theoretic measures, which imply that the only slightly better fit in equation (4) does not warrant the additional parameter. Similarly, we reject fitting $W$ to the data and instead use an a priori physical basis for setting its value. Figure 2 shows the best-fit value to the strike-slip events of $\Delta \sigma=3.91 \mathrm{MPa}$, assuming $W=15 \mathrm{~km}$ and $\mu=30 \mathrm{GPa}$, shown with a solid black line.

\section{Other Focal Mechanisms}

For dip-slip faults, two effects influence the scaling. One effect is that the downdip width $W$ is generally larger for dipping faults. For a seismogenic depth $H$ and fault dip $\theta$

$$
W=H / \sin \theta .
$$

(Later, we will generalize this slightly to consider the potential for ruptures to extend below the seismogenic layer.) For dips of $90^{\circ}$ (strike-slip), $60^{\circ}$ (normal), and $30^{\circ}$ (thrust), the factor $1 / \sin \theta$ is, respectively, $1,1.15$, and 2 . Thus, only for quite shallow dips is this a significant factor. A second factor is a change in the stiffness coefficient on $W$ for the infinite rupture, with the factor of 2 replaced by the Lame parameter ratio $(\lambda+2 \mu) /(\lambda+\mu)=1 /\left(1-\left(V_{S} / V_{P}\right)^{2}\right)$ where $V_{S} / V_{P}$ is the ratio of the shear wave to that of the compressional-wave speed. Using typical values of this wave-speed ratio of 1.75 yields typical values of $\sim 1.5$ for this factor or $\sim 3 / 4$ of the value for dip-slip relative to that of strike-slip. Thus, for dip-slip cases, we have

$$
S=\frac{\Delta \sigma}{\mu} \frac{1}{\frac{7}{3 L}+\frac{1}{\left(\frac{\lambda+2 \mu}{\lambda+\mu}\right) W} .}
$$

We can further generalize this to the oblique slip case for slip in the arbitrary direction $\hat{s}$ consisting of along-strike and dip-slip components:

$$
S=\frac{\Delta \sigma}{\mu} \frac{1}{\frac{7}{3 L}+\frac{1}{W \hat{s} \cdot\left(2, \frac{\lambda+2 \mu}{\lambda+\mu}\right)}}
$$

or equivalently

$$
S=\frac{\Delta \sigma}{\mu} \frac{1}{\frac{7}{3 L}+\frac{1}{W \hat{s} \cdot\left(2, \frac{1}{1-\left(V_{S} / V_{P}\right)^{2}}\right)} .}
$$

Figure 2 shows these combined effects for the three dip cases, of $\theta=90^{\circ}$ using equation (3), and $\theta=60^{\circ}$ and $30^{\circ}$ using equation (7). We assume constant seismogenic depth and correspondingly varying downdip $W$, and include the modulus effect in equation (7) and typical values for the moduli for the two dip-slip cases. We see the modulus effect outperforming the increased $W$ effect at $60^{\circ}$, so the expected slip is slightly lower for the $60^{\circ}$ dip case. The crossover where the two effects are comparable occurs at $\sim 45^{\circ}$. By $30^{\circ}$, the increased $W$ effect dominates and slip is indeed increased, but not by a large amount. It is not until we get to faults with a very shallow dips that an entirely new scale of slip occurs, at dips and $W$ 's relevant to subduction zones. Thus, for subareal surface-rupturing events under 


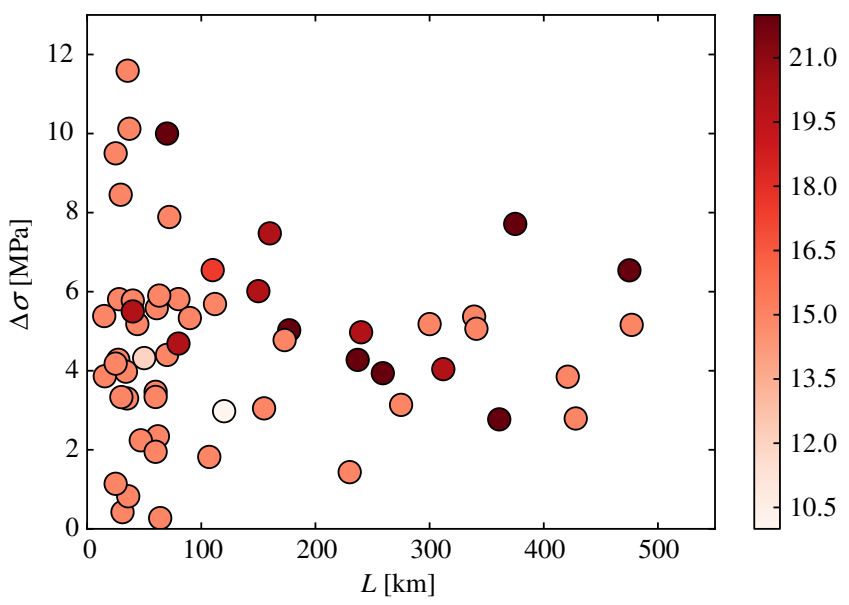

Figure 3. Stress drop as a function of $L$. A constant $W=15 \mathrm{~km}$ is assumed for width in the calculation. Shades of symbols represent values of $W$ in $\mathrm{km}$ in the (Wesnousky, 2008) database. Assuming constant $W$ presumes the least knowledge about uncertain downdip widths. A slightly better fit, in terms of slightly reduced scatter, is obtained using the variable widths in the database. However, this shows that even just with surface data, we get relatively small scatter. The color version of this figure is available only in the electronic edition.

constant stress-drop scaling, we do not expect vastly different slip values for dip-slip versus strike-slip events. And the data, although sparse, appears to support this.

\section{Constant Stress-Drop Values}

Given the success of the constant stress-drop scaling, further plots are warranted. Figure 3 shows inferred values of individual stress drops, using equation (3) and data for slip and length scales. In the figure, we use a constant $W=15 \mathrm{~km}$, since this allows stress-drop estimates to be derived from surface data alone. Using the $W$ 's from the database as opposed to a constant value of $W=15 \mathrm{~km}$ appears to improve the estimates slightly, reducing the coefficient of variation and residual $W$ dependence somewhat. Assuming $W=15 \mathrm{~km}$, and specifically examining large earthquakes so that $L \geq 15 \mathrm{~km}$, the $\mathrm{COV}$ (coefficient of variation equal to the standard deviation divided by the mean) is 0.430 . Using variable $W$, the COV is 0.416 . Note that these COVs are remarkably small. These factor-of-2 variations compare with closer to factor-of-10 variations on either side of the mean values at small events (Hanks, 1977). One explanation could be that measurement uncertainties in corner frequencies affect the scatter at small events, more than measurement uncertainties in $L$ and $W$ for large events. Or it could be a real effect.

Interestingly, the mean value for the stress drop inferred from the large event surface-slip data is remarkably consistent with mean values inferred seismologically from small events (Hanks, 1977; Allmann and Shearer, 2009) and from aftershock distributions (Rubin, 2002). While there are substantial uncertainties associated with mean values at small events due to inferences about corner frequencies (for example, Brune [1970] scaling versus Madariaga [1976] gives a factor-of-5 difference in seismologically determined average static-stress drop), the consistency of constant stress-drop scaling across the entire range of magnitudes is a remarkable observation.

We next examine how to use slip-length scaling in seismic hazard analysis.

\section{Magnitude-Area Scaling and Implied Slip-Length Scaling}

As noted in the Introduction, magnitude-area scaling relations have been employed two ways in hazard estimates, one to estimate sizes of events and the other to estimate rates. However we choose to apply constraints to estimate rates, estimating sizes from magnitude-area relations remains the most accurate method. We thus begin with magnitude-area scaling relations.

We examine four magnitude-area scaling relations here. Two are the branches of a recent hazard analysis for California (WGCEP, 2008), the third generalizes one of these relations (Shaw, 2009), and the fourth generalizes a long standing relation (Wells and Coppersmith, 1994). The first is Ellsworth-B (2003; EB):

$$
\mathbf{M}=\log A+4.2 .
$$

This has the virtue of being extremely simple, as a oneparameter fit, and being a good empirical fit to the large earthquake ( $\mathbf{M}>6.5)$ data for which it was developed. The second is Hanks and Bakun (2002; HB02), a two-regime scaling:

$$
\mathbf{M}= \begin{cases}\log A+3.98 & A \leq 537 \mathrm{~km}^{2} \\ \frac{4}{3} \log A+3.07 & A \leq 537 \mathrm{~km}^{2} .\end{cases}
$$

This has the virtue of more accurately fitting the data at smaller magnitudes. The third is Shaw (2009), which is a generalization of the Hanks and Bakun model; it extends the bilinear Hanks-Bakun (HB) two-regime scaling to that of a three-regime scaling, with a third asymptotic regime valid for very long ruptures $L \gg W$ whereby $S$ approaches $W$ scaling asymptotically. This is done at the price of one additional scaling parameter (the length scale at which the transition to the third regime occurs). The Shaw (2009; S09) scaling relation is parameterized as

$$
\mathbf{M}=\log _{10} A+\frac{2}{3} \log _{10} \frac{\max \left(1, \sqrt{\frac{A}{W^{2}}}\right)}{\left(1+\max \left(1, \frac{A}{W^{2} \beta}\right)\right) / 2}+\text { const. }
$$

Here, $W$ is the rupture width, and $\beta$ is a fitting parameter that gives a crossover scale length to the asymptotic $W$ scaling. To match the small events, the constant is set to be the same as in the HB relation: const $=3.98$. In the limit of $\beta \rightarrow \infty$, the scaling relation reduces to the $\mathrm{HB}$ scaling relation and, in this sense, represents a one-parameter extension, if one chooses to 
(a)

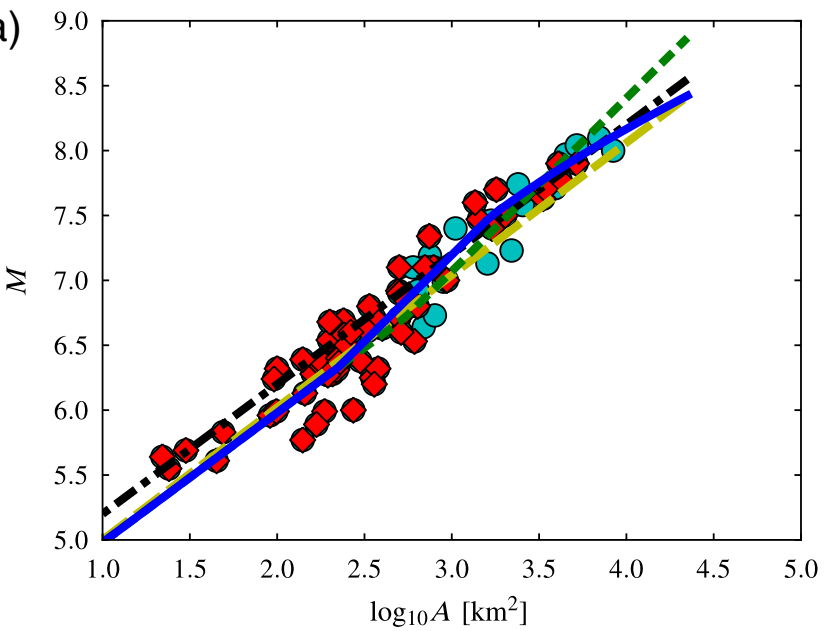

(b)

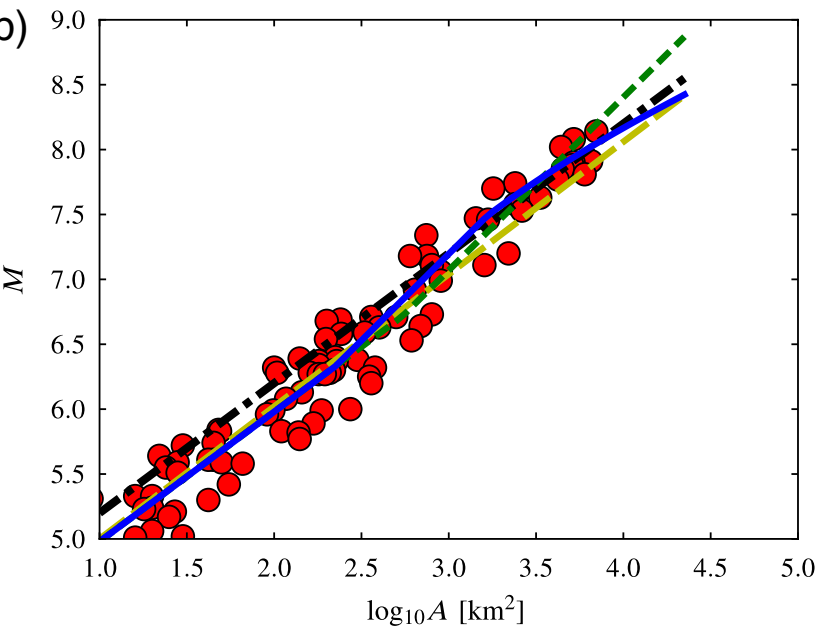

Figure 4. Magnitude area relations for large strike-slip events. (a) WGCEP03 data. Dark circles denote $W<15 \mathrm{~km}$ events, and light circle denote $W>15 \mathrm{~km}$ events. (b) Circles denote magnitude and area of events from Hanks and Bakun (2008) database. The dash-dot line is the linear Ellsworth-B (WGCEP, 2003) magnitude-area relation, equation (10). Short-dash line is the Hanks and Bakun (2002) bilinear relation, equation (11). The long-dashed line is the Wells and Coppersmith (1994) scaling relation, equation (13). The solid line is the Shaw (2009) scaling relation, equation (12). The S09' relation is illustrated using default $W$ values, with $W=15 \mathrm{~km}$. The color version of this figure is available only in the electronic edition.

treat $W$ as a parameter. Note also that in the limit as $L \rightarrow \infty$, it scales asymptotically as $\mathrm{M} \sim \frac{2}{3} \log _{10} A$, which gives $S \sim W$, as the Starr (1928) and Knopoff (1958) solutions suggest. Appendix $\mathrm{B}$ discusses how the parameters in this scaling relation are determined.

The fourth is a Wells-and-Coppersmith-type linear relation between $\mathbf{M}$ and $\log _{10} A$

$$
\mathbf{M}=C_{1} \log _{10} A+C_{0} .
$$

The EB relationship is a special case of these linear relationships, with $C_{1}=1$. Here, the additional parameter introduces new scaling physics. Allowing for a nonspecific parameter on the area term, as the Wells-Coppersmith fitting does, adds substantial complexity to the model, given that it is an exponent on the area. Progressing from a discrete small set to a continuum is a leap in dimension, which would require a much better fit to be worth generalizing in such broad terms.

\section{Magnitude-Area Data}

Next, we compare the scaling relations with the data. A number of databases were used, and they were chosen due to their published availability and use in vetted seismic hazard estimates. Because of concerns about the potential influence of downdip width, the WGCEP03 database containing magnitude, length, and width was used (WGCEP, 2003) to calibrate width-sensitive fits, in particular the S09 equation (12) relation. The WGCEP03 database is mainly a subset of the Wells and Coppersmith (1994) data, selected for quality control. (The data are reproduced in (E) Table S1, in the electronic supplement.) The Hanks and Bakun (2008) magnitude-area database was used as another target, providing some additional large events and alternative interpretations of areas, par- ticularly at small magnitudes. (The data are reproduced in (E) Table S2, in the electronic supplement.)

One limitation of these databases is a lack of explicit error estimates for the data. Wells and Coppersmith (1994) have a good discussion of the wide range of issues generating uncertainties in the data. One approach, developed by Ellsworth (2003), is to use the variability of published estimates from similar events to obtain an empirical estimate of data uncertainties. This leads to the not obvious result that area estimates are substantially more uncertain than magnitude estimates ( 0.25 in $\log _{10}$ area versus 0.08 in magnitude). At the same time, many of the scaling relations were optimized using least-squares regressions, which implicitly neglect errors in the horizontal axis. A useful extension is the orthogonal distance regression (ODR), which explicitly accounts for data uncertainties in the horizontal and vertical directions. A beneficial feature of ODR is that in the limit of the data uncertainties on the vertical axis being large compared with the data uncertainties on the horizontal axis, they converge to leastsquares estimates. Moreover, when data uncertainties are not precisely known, erring on the side of larger estimates in the vertical axes relative to that of the horizontal gives a better estimate of parameter uncertainties (Boggs and Rogers, 1990). To best compare against previous scaling relations and to operate in a mode useful for hazard estimates, we use leastsquares error metrics to compare all models, in addition to ODR estimates with large vertical data uncertainties relative to that of horizontal to estimate parameter uncertainties.

Figure 4 shows fits of the scaling relations to the data. Deviation on this $\log -\log$ scale can be observed, but statistical testing helps to illuminate things further as we will discuss now. Table A1 in Appendix A shows standard deviations of the fits relative to the data, with the models listed in rank order based on AIC (Akaike, 1974), which rewards 
minimizing errors while penalizing for extra parameters; minimum AIC is best. AIC $=-2 \ln \mathcal{L}+2 k$ where $\mathcal{L}$ is the likelihood and $k$ is the number of fitted parameters. Thus, differences in AIC correspond to differences in loglikelihood, with relative likelihood of two models being $e^{-\Delta \mathrm{AIC} / 2}$. This gives a scale of the differences in AIC that matter, at least a few to be significant. AIC is similar to Fisher $F$-test in that they both examine the importance of the fit relative to the cost of extra parameters. AIC has an advantage in that it does not require the lower parameter model to be embedded in that of the higher. Used together, standard deviation and AIC provide a helpful view of differences in fits: differences in standard deviation give relative variations in how well the curves are fitting in an absolute sense. Differences in AIC offer a sense of how statistically significant, in a log-likelihood sense, the differences are.

With regard to data fits, one concern involves whether fits across the entire magnitude range or only at large events should be considered. There are substantial uncertainties in values of area for the small events; however, these events also are used in hazard calculations, so matching the scaling in that regime is important. Both sets of error measures, across the entire magnitude range, and just for the large events, are therefore shown in the table.

Table A1 (a) in Appendix A shows fits to the WGCEP03 data. Table A1 (b) in Appendix A restricts the fits to $\mathbf{M}>6.5$. Table A1 (c) in Appendix A shows the HB data, and Table A1 (d) in Appendix A the fits to $\mathbf{M}>6.5$ for that data. Because only area values are given in the HB database, the S09 relation fixes $W$ as a parameter in fitting that data.

The HB and S09 relations work best across the entire magnitude range, while that EB and S09 work best specifically for the large events. The original Wells-Coppersmith relation (WC in the table) somewhat underpredicts the HB dataset at the large events. Refitting the parameters, we find best fitting $C_{1}=1.08$ and $C_{0}=3.86$ ( $\mathrm{WC}^{\dagger}$ in Table A1 in Appen$\operatorname{dix} \mathrm{A})$. This does a better job, but as we will observe when examining other types of data, the nonrobustness of the fits (in particular, changing $C_{1}$ for other different datasets) raises issues for this functional form. When width information is available, the S09 relation using fault based $W$ (denoted S09 in the tables) does better than using fixed values of $W$ (denoted S09' in the tables), so this is the preferred version of that relation.

Comparing fits of the different scaling relations across the entire range of magnitudes $(\mathbf{M}>5)$ the S09, HB, and modified WC-type scalings fit better than the EB scaling. For only the large events, $(\mathbf{M}>6.5)$ however, which the EB relation was developed to match, it does quite well. Using as an error metric the difference in magnitude, Fisher $F$-tests support what we observe in AIC: from the lowest magnitudes $(\mathbf{M}>5)$, the $\mathrm{S} 09$ relation fits better than that of the $\mathrm{EB}$, as does the $\mathrm{HB}$ relation relative to that of the EB. But for large magnitudes $(\mathbf{M}>6.5)$, the EB relation begins to perform well. At the large magnitudes, the EB, S09, and modified WC relations are comparable. In contrast, the $\mathrm{HB}$ relation begins to perform less accurately. For the entire magnitude range, the S09 relation fits better than the HB relation, significantly in the WGCEP03 data where width information is available, although only just above the level of statistical significance in the HB data where width data is unavailable and default $W$ values must be used. As the magnitude cutoff increases, the S09 relation does even better against the HB relation. Thus, the extra regime in S09 relative to $\mathrm{HB}$ appears justified in terms of a better statistical fit.

Substantial differences in the scaling relations exist in the $\mathbf{M} 6$ range, a range that is significant from the point of view of hazard for many areas. Indeed, moderate magnitude events can often dominate the hazard; while they are much smaller than the large events, they also are much more frequent, and thus more likely to occur nearby. Proximity is a major component of hazard, as the recent 2010-2011 events in Christchurch, New Zealand, graphically illustrate. Distinguishing between the different scaling relations in the $\mathbf{M} 6$ range will not be helped by our slip-length scaling approach here and will have to be dealt with using other methods. (Finite source inversions and precise relative relocations of aftershocks are two potential methods of pursuing these issues.) Substantial differences also exist at the largest events. Vastly different scalings are proposed for the three different relations, with moment $\mathcal{M}$ scaling as $\mathcal{M} \sim A^{2}$ in $\mathrm{HB}, \mathcal{M} \sim$ $A^{3 / 2}$ in $\mathrm{EB}$, and $\mathcal{M} \sim A$ in S09. Distinguishing between these different scalings in magnitude log area space is difficult to accomplish for the limited dataset available at the largest magnitudes. When we seek to examine summed constraints, however, these differences become significant and measurable, a subject to which we now turn.

\section{Implied Slip-Length from Magnitude Area}

Here, we transform magnitude-area scaling into a corresponding implied slip-length scaling. Satisfying integral constraints by moment balancing, or, as we argue for in this paper, slip-rate balancing, the slip from each event is summed to obtain an overall rate of events to match the longterm slip rate on the faults. This slip is summed linearly; as such, it makes sense to determine how the scaling relations look not in log-log space, which emphasizes the range of sizes, but in linear-linear space, which emphasizes the largest events that are dominating the sum. To convert the magnitude-area scaling relations to implied slip-length scaling relations, we use the same assumptions involved in performing moment balancing. We convert magnitude $\mathbf{M}$ to moment $\mathcal{M}$ and divide by area $A$ and modulus $\mu$ to get slip $S$ : $S=\mathcal{M} / A \mu$. A standard value of the modulus is used $(\mu=30 \mathrm{GPa})$, an issue to which we will return when we subsequently discuss how the magnitude-area data compares with the surface-slip measurements. In converting moment to slip, we can see the uncertainty in the downdip width playing out: if our area estimates are biased, our slip estimates will be biased. For length and width, we can use the information in databases directly when it is available, as in the WGCEP03 data. Figure 5 a shows this transformed data. When it is 

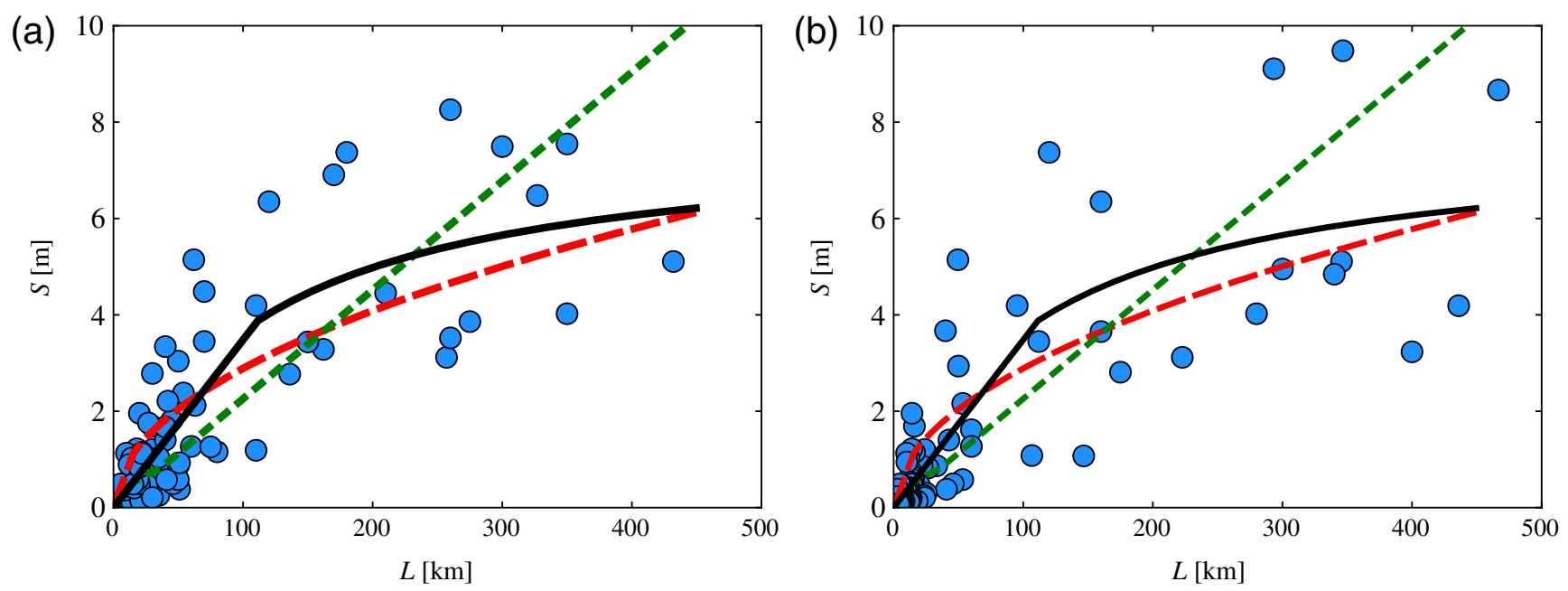

Figure 5. Fits of implied slip-length scaling by magnitude-area scalings. Magnitude is converted to moment, then divided by area and modulus to get slip. Length is used when it is available in the database. Otherwise, area is converted to length by dividing area by width, assuming seismogenic depth $H=15 \mathrm{~km}$; data are shown with circles. Different line styles represent different magnitude-area scaling relations rescaled in the same way the data has been. Parameters used directly from magnitude-area scaling relations, Table A2 (a) in Appendix A. The curves are the implied slip-length for Ellsworth-B (WGCEP, 2003) (long dash), Hanks-Bakun (Hanks and Bakun, 2008) (short dash), and Shaw (2009) (solid). The S09' relation is illustrated using default $W$ values, with $W=15 \mathrm{~km}$. (a) Data derived from WGCEP03 database. Event specific $W$ 's used. (b) Data derived from Hanks-Bakun database. The default of $W=15 \mathrm{~km}$ was used. The color version of this figure is available only in the electronic edition.

unavailable, as in the HB data, we can divide the area by downdip width $W$ to get length $L: L=A / W$. Figure $5 \mathrm{~b}$ shows this transformation assuming $W$ equals $L$ for small events, but then saturates for large events at the downdip width $W$. That is, $W=W^{*}$ where

$$
W^{*}=\min (L, W)= \begin{cases}L & L \leq W \\ W & L>W\end{cases}
$$

with typical values of the seismogenic width $W=15 \mathrm{~km}$ for vertical strike-slip faults, and downdip widths for dipping faults having correspondingly larger values. Assuming a default downdip width, as in equation (14), we can infer an $L$ from $A$. Alternatively, individual values of $L$ from compiled databases (e.g., Wells and Coppersmith, 1994) can be used directly with the HB data. We have also determined that when we use individual values of $L$ associated with the events, we get similar results, whether we assume a default value for $W$ and derive an implied $L$, or we use individual values of $L$. While individual points do move to some degree, the overall trend is similar. Because of the simplicity and clarity associated with using implied length in conjunction with implied slip, we focus our attention on that approach when using the HB data.

Again, assuming $W=W^{*}$ the magnitude-area scaling relations transform into slip-length scaling relations as follows. For EB, we get

$$
S \sim A^{1 / 2}=C_{2}\left(L W^{*}\right)^{1 / 2}= \begin{cases}C_{2} L & L \leq W \\ C_{2}(L W)^{1 / 2} & L>W\end{cases}
$$

For generalized WC-type scaling, we get

$$
\begin{aligned}
S \sim A^{1.5 C_{1}-1} & =C_{3}\left(L W^{*}\right)^{1.5 C_{1}-1} \\
& =\left\{\begin{array}{ll}
C_{3} L^{3 C_{1}-2} & L \leq W ; \\
C_{3}(L W)^{1.5 C_{1}-1} & L>W
\end{array} .\right.
\end{aligned}
$$

For HB, we get

$$
\begin{aligned}
S & \sim \begin{cases}\left(\frac{A}{537}\right)^{1 / 2} & A \leq 537 \mathrm{~km}^{2} ; \\
\frac{A}{537} & A>537 \mathrm{~km}^{2}\end{cases} \\
& = \begin{cases}C_{4} L & L \leq W ; \\
C_{4}(L W)^{1 / 2} & W<L<537 \mathrm{~km}^{2} / W ; \\
C_{4} L W /(537)^{1 / 2} & 537 \mathrm{~km}^{2} / W \leq L\end{cases}
\end{aligned}
$$

For S09, we get

$$
S \sim \frac{2 A / W}{1+\max \left(1, A / W^{2} \beta\right)}= \begin{cases}C_{5} L & L \leq W \beta \\ \frac{2 C_{5}}{L}+\frac{1}{W \beta} & L>W \beta .\end{cases}
$$

Taking into account the crossover effects of the finite seismogenic width expressed in equation (14), we find the magnitude-area scaling relations transformed into that of slip-length scaling are modified to have an additional regime for the EB, generalized WC, and $\mathrm{HB}$ scaling relations, while the $\mathrm{S} 09$ scaling relation is instead reduced by one regime and one parameter, from three to two regimes, and from three to two parameters $\left(C_{5}\right.$ and $W \beta$ in equation 18). This reduction for the $\mathrm{S} 09$ scaling relation is not coincidental because it was developed from a slip-length scaling relation; that slip-length scaling relation is a generalization introduced by Manighetti et al. (2007) of a scaling relation by Shaw and Scholz (2001).

Comparing the different scalings for large $L$, we again note very different predicted behaviors. For $L \gg W$ in HB $S \sim L$, in Ellsworth-B $S \sim L^{1 / 2}$, and in S09 $S \sim W$. In the 
generalized WC scaling, $S \sim L^{1.5 C_{1}-1}$, a nonspecific exponent. The scalings also differ in their $W$ dependence at large $L$, with a linear dependence on $W$ in $\mathrm{HB}$ and $\mathrm{S} 09$, and a $W^{1 / 2}$ dependence in EB. In the generalized WC scaling, $S \sim W^{1.5 C_{1}-1}$, again, a nonspecific exponent. Unfortunately, the $W$ values for the data are too uncertain to test the various predicted dependences. Fortunately, the data does appear good enough to test some features of the $L$ dependence.

Examining the fits of the transformed scaling relations to the transformed data in Table A2 in Appendix A, the S09 relation is seen to be the best, followed closely by $\mathrm{EB}$, then the modified WC and then HB. Figure 5 shows the fits to the data.

Finally, the answers are robust with respect to uncertainties in the data. We have also fit using ODRs, which explicitly account for uncertainties in the data. A variety of estimates of the data uncertainties were examined, including estimates derived from data by Ellsworth (WGCEP, 2003). These alternative fits changed parameters by typically only a few percent up to a few tens of percent and preserved the conclusions based on relative fits.

\section{Surface Slip}

\section{Surface Slip Data}

In the previous section, we showed how transforming and reweighting magnitude-area scaling relations helped to test them against data they were developed to fit, and to better match the scaling relations to summed integral constraints. Surface-slip data enables us to proceed far beyond this, however, by bringing in independent data. With independent data, we can validate the scaling relations. Surface-slip data brings a number of advantages, as well as disadvantages. One of the greatest advantages is that for large subareal events, it is geologically observable and provides a ground-truth, modelindependent measure of the earthquake source. A disadvantage is that it is only a slice, a view at the surface that requires interpretation to infer what occurs at depth. It is also an imperfect recorder, as noise in the system (Shaw, 2011) and comparisons with sophisticated geodetic measurements (Wei et al., 2011) make clear. An important source of uncertainty in surface-slip measurements is the practice of enveloping the data, with slip minima along the surface rupture often removed in calculating geologically estimated average slip values (Wesnousky, 2008).

Using surface-slip data to constrain rates has a number of advantages despite the uncertainties in the data. First, it manages to avoid some of the uncertainty surrounding the issue of the depth of rupture in large events. Second, it introduces into the analysis a new, independent geologically observable dataset, thereby bringing in additional testable and collectible information to the estimates. Third, by using the linear measure of slip rather than the logarithmic measure of magnitude as the independent variable, fits to the scaling relations are more naturally mapped onto the linearly summed integral constraints, so errors are more accurately accounted for where they matter most. Finally, it allows for the ability to incorporate pre-instrumental information, opening up the possibility to vastly extended datasets.

\section{Surface-Slip Scaling Relations}

Here, we simplify the slip-length scaling relations. Generally, only large earthquakes, events which break the full seismogenic thickness, tend to break the surface and show significant slip there. For strike-slip events, we are therefore referring to $L \geq W$ or $L>15 \mathrm{~km}$ and $M \geq 6.5$ events. Thus, rather than having the added complication of the two-regime $W^{*}$ in equation (14), we can reduce to a simpler case with only the saturated $W$ regime. Carrying over the scaling relations from the magnitude-area scaling, and simplifying the multiple regime scaling to those which matter in the large-event sliplength scaling, we determine four candidate scalings.

The first is a square root $L^{1 / 2}$ scaling consistent with the implied EB scaling:

$$
S \sim A^{1 / 2}=C_{6}(L W)^{1 / 2} .
$$

This kind of scaling has been proposed by Leonard (2010) for large lengths. Wesnousky (2008) also considered an $L^{1 / 2}$ scaling for slip, although not its $W$ dependence. The second is a linear $L$ scaling consistent with the implied HB scaling for the largest events,

$$
S \sim A=C_{7} L W .
$$

The third is a power-law scaling, consistent with the generalized WC-type scaling. This adds an extra free parameter, a nonspecific exponent $\eta$ in the scaling, so a much better fit will be required to make the added model complexity and potential physics worthwhile:

$$
S \sim A^{\eta}=C_{8}(L W)^{\eta} .
$$

For the fourth scaling, we replace the two-regime implied slip-length scaling with the new constant stress-drop scaling (S12), equation (3), derived earlier. They come from a similar approach, but the constant stress-drop scaling has the advantage of being a reduced parameterization. For ease of discussion, we reproduce it here:

$$
S=\frac{\Delta \sigma}{\mu} \frac{1}{\frac{7}{3 L}+\frac{1}{2 W}} .
$$

\section{Comparing Fits to Surface Slip Data}

Which scaling relation best fits the surface slip data? We have two different types of scaling relations to compare with the data: the implied slip-length data from the magnitudearea scaling, equations (15)-(18); and the simplified surface slip-length scalings, equations (19)-(22). Note that the two sets of scalings are addressing different things, and while they should agree asymptotically for the largest events, the details at the smaller events differ. Therefore, we would 

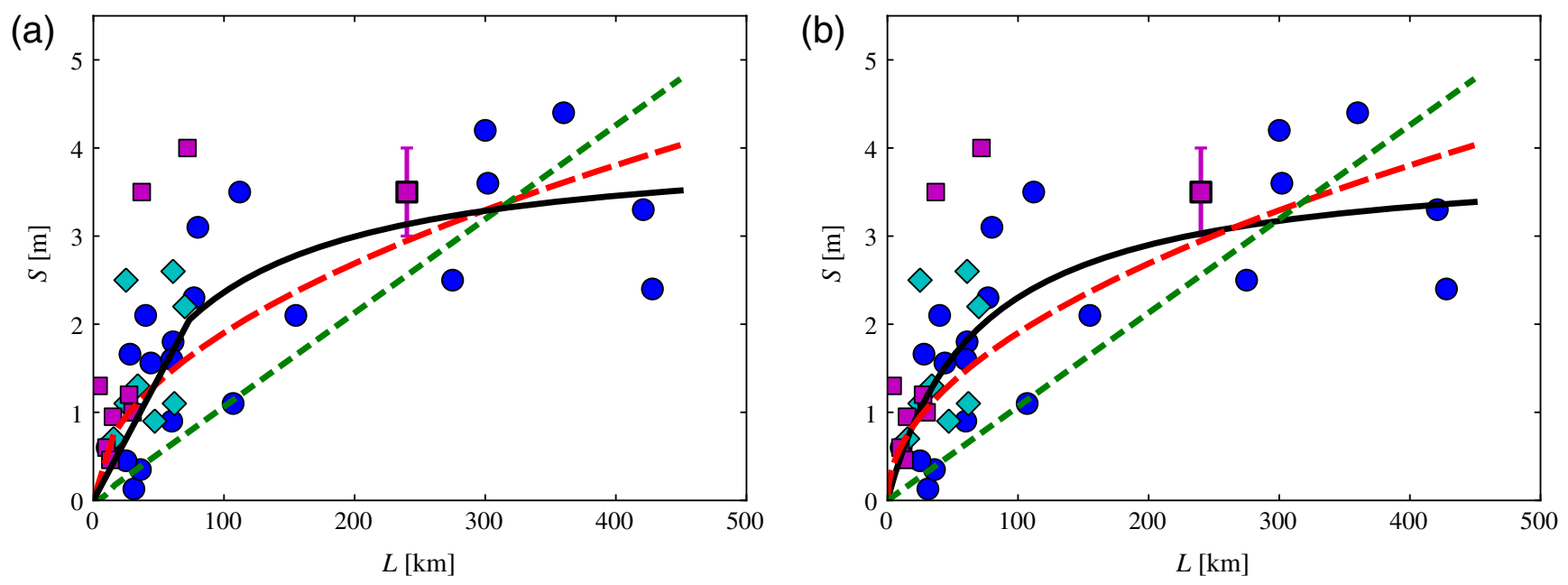

Figure 6. Fits of various scaling relations to surface slip observations of average slip versus length. Symbol types indicate focal mechanisms: strike-slip (circle), normal (diamond), and thrust (square). (a) Implied slip-length from magnitude-area scaling fits of surface-slip data. In order of best fit to least good fit: solid line shows equation (18) S09 scaling; long-dashed line shows equation (15) Ellsworth-B (EB) scaling; short-dashed line shows equation (17) Hanks-Bakun (HB) scaling. (b) Surface slip-length scaling fits of surface-slip data. In order of best fit to least good fit: solid line shows equation (22) constant stress-drop scaling; long-dashed line shows equation (19) $L^{1 / 2}$ scaling; shortdashed line shows equation (20) $L$ scaling. Note that the two types of scaling relations, the implied slip-length curves in (a) and the surface slip-length curves in (b) differ only at the smaller length scales, and provide comparable-looking fits. Note the change of scale relative to Figure 5. The color version of this figure is available only in the electronic edition.

expect the different sets to behave best on the data they were developed to address.

Figure 6a shows the fits of equations (15)-(18), and Figure $6 \mathrm{~b}$ shows the fits of equations (19)-(22) to the surface-slip data. The two different sets are seen to fit comparably, with the detailed differences at the smaller $L$ values not making much difference to the overall fit; indeed, the two plots are difficult to distinguish. We observe only small differences between scaled fits of the implied slip-length scaling from magnitude-area scaling relations and the simplified surface-slip scalings for this surface-slip data, which is mostly dominated by larger events where the two sets of relations are similar. Standard deviations are (see Table A3 in Appendix A), respectively, 1.174 for linear $L$ scaling, 0.804 for square root $L^{1 / 2}$ scaling, 0.760 for equation (22) S12 scaling, and 0.741 for equation (18) S09 scaling. Accounting for free parameters, the additional parameter in equation (18) scaling is not justified, and by an AIC criteria, the S12 fits best. Details are reported in Appendix A. ODRs explicitly accounting for data uncertainties also yield similar answers.

One important thing to notice in Figure 6 relative to Figure 5 is the change in scale on the vertical axis. While the functional forms of implied slip-length scaling relations perform reasonably well in fitting both the implied sliplength data in Figure 5 and the surface slip-length data in Figure 6a, there is a significant change in the overall amplitude of the slip, with noticeably less slip in the surface-slip data. We will return to a discussion of this shortly, when we examine reconciling differences in the slip estimates.

Regarding the power-law fit of the generalized WC-type relation, two features of this fit are a concern for this functional form. First, the best fitting exponent differs substantially for the implied slip and surface-slip datasets, so we are not getting stable results. Second, the uncertainties in the amplitude value $C_{7}$ are very large relative to the values themselves, again pointing to the sensitivity in parameter fits to the data. Simply put, this functional form is not a good parameterization of the data. These results also may help to explain the large uncertainties found by Wells and Coppersmith (1994) in fitting their regressions to surface-slip data, and the variable exponents found in fitting the generalized WC-type regressions to regional tectonic data (e.g., Yen and Ma, 2011).

\section{Reconciling Differences in Magnitude-Area and} Surface Estimates of Slip

Ideally, estimates of average slip from magnitude-area and slip-length data would agree. However, there are some systematic differences in the estimates. As noted earlier in comparing Figure 5 with Figure 6, magnitude-area estimates of average slip were found to be larger than surface-slip estimates. Attempting to reconcile the estimates, we note one difference is that different events are considered in the two databases. To control for this, we compare events which are common to both datasets. (The data are reproduced in (E) Table S4, in the electronic supplement.) Examining the ratio of the surface slip estimate to the magnitude-area estimate, shown in Figure 7, we find the magnitude-area estimate assuming $W=H=15 \mathrm{~km}$ is on average roughly $30 \%$ more (average ratio $S /(\mathcal{M} / A \mu)=0.75$ for data points in the figure). We find, as well, no obvious magnitude dependence to this difference. Wells and Coppersmith (1994) also noted a 


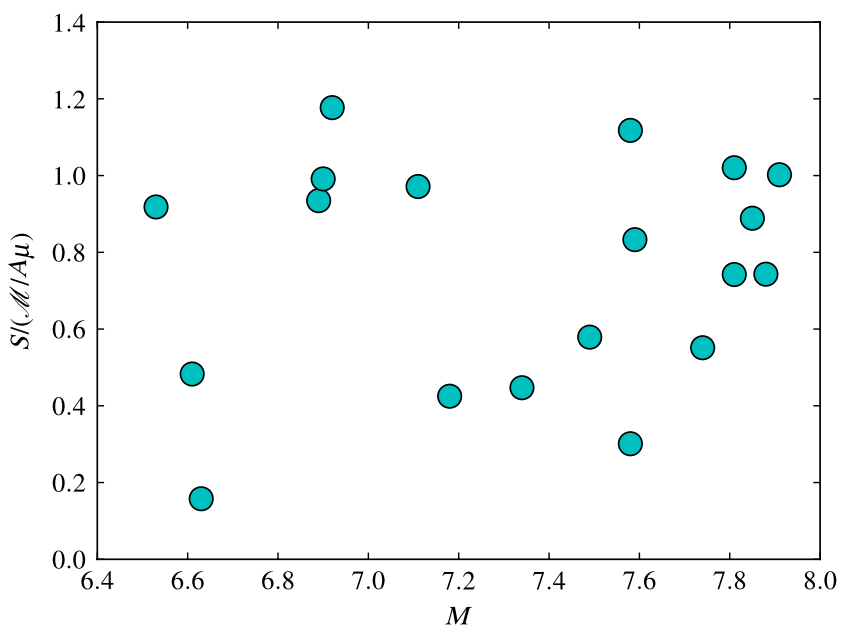

Figure 7. Exploring discrepancy between implied average slip values from magnitude-area and surface-slip data. Ratio of data in Figure 6 to data in Figure 5b for same events in both plots. Surfaceslip measurements average $\sim 30 \%$ lower than magnitude-area estimates. The color version of this figure is available only in the electronic edition.

similar discrepancy in slip estimates from surface and depth measurements. Two possibilities arise in understanding this. One is that the surface-slip measurements are systematically low, missing some slip. The other is that the magnitude-area estimates are systematically high, mapping specific deep coseismic slip below the seismogenic layer onto the seismogenic layer. Indeed, adopting the best fitted $W=19 \mathrm{~km}$ from the S09 fit to the magnitude-area data mostly resolves the discrepancy. Both of these possibilities appear to be valid concerns and are a source of genuine uncertainty.

Related to the question of moment estimates potentially being biased high, we need to divide by the modulus to connect from moment to slip. We used a uniform value of $30 \mathrm{GPa}$ for all events. This is a standard value used, but others are also sometimes used. For example, using $33 \mathrm{GPa}$ as an alternative value would reduce the moment-estimated slip by $10 \%$, reducing the average discrepancy with the surface measurements from $\sim 30 \%$ to $20 \%$. Wesnousky (2008) also discusses this question about modulus impacts on moment estimates. Modulus uncertainties should to be taken into account in slip-rate estimates from magnitude-area scaling relations. It is an additional advantage of surface-slip measurements that they do not involve this quantity-and thus, uncertainties in this quantity-in slip-rate estimates.

Regarding the possibility that geological measurements of surface offset may be underestimating true offset, recent technological advances have allowed for new views of this question. For the 2011 M 7.2 El Mayor-Cucapah earthquake, Wei et al. (2011) combined before and after LiDAR pictures mapping vertical offsets with before and after optical pictures mapping horizontal offsets providing an image of unprecedented detail and fidelity of complete, near-field fault displacements and offsets. A clear outcome of that data, combined with the research of Hudnut et al. (2010) was that envelopes of geologically measured surface offsets better represented the true offsets than simple averages, with the geological offsets often missing displacements observed in high-resolution geodetic imaging. The work on the El Mayor-Cucapah event is the most accurate view, but it remains just one event for now.

Support from an ensemble point of view that slip at the surface may not all be recorded comes from a statistical analysis of surface displacements. Shaw (2011) analyzed differences in surface slip as a function of separation and found substantial average differences in a slip of $1 \mathrm{~m}$, down to the smallest separations. At the same time, maximum slips were well bounded, thus it is not so much an added as it is subtracted noise to underlying slips. Missing slip due to unconsolidated near-surface layers is the simplest explanation of these results. Uncertainties in the enveloping procedures used to estimate average slip at the level of tens of percent remain and is an unresolved uncertainty.

Finally, we can use averages in the differences between implied slip length from magnitude-area and surface slip length to separate the effects from different catalogs used. Ratios of the fit parameters of the two leading scaling relations $L^{1 / 2}$ and $S 12$ for the surface-slip relative to that of the impliedslip dataset give, from Table A3 (b) relative to Table A2 (c) in Appendix A, values of $\sim 0.65$. Dividing this by a factor 0.75 from the systematic difference for the same datasets gives an $\sim 15 \%$ effect due to the different databases. The main difference in the databases is in how a number of events included in the magnitude-area database are not present in the slip-length database. A significant number of much older first half of the 20th century events in Tibet and Mongolia account for much of this difference. Regressions that included these events would thus be expected to raise amplitudes of fits to surface data of slip-length scaling relations by $\sim 15 \%$.

\section{Deep Slip}

The possible existence of significant amounts of coseismic slip below the seismogenic layer in the deeper, stably sliding fault remains an open question as yet unresolved by observations. Dynamic modeling results suggest it is a real possibility, but parameter uncertainties and particularly observational resolution uncertainties make this difficult to constrain, and, as such, remains an unresolved uncertainty.

Modeling results show a significant fraction of slip may be driven coseismically below the seismogenic layer, on the order of a third of the total moment (Shaw and Wesnousky, 2008). This slip occurs mainly as long-period motion, with a dearth of high frequencies (Shaw and Wesnousky, 2008), and thus occurs in moment and magnitude estimates, but not necessarily in that of shaking. Moreover, it may be misplaced in inversion algorithms which map slip onto sources of higher frequency radiation. This may be one reason synthetic ground-motion simulations have had difficulty reproducing empirical ground-motion prediction and standard 
magnitude-area scaling relations simultaneously (Graves et al., 2011).

Reconciling average surface slip with that of magnitudearea estimates thus remains an unresolved uncertainty. The surface-slip estimates may be biased a bit low due to the missing slip; or the magnitude-area estimates may be biased a bit high due to mapping of deep slip onto shallower seismogenic depths. While this uncertainty remains, there are nevertheless two things we can report. First, the functional forms of slip-length scaling observed geologically and inferred from the magnitude-area scaling are similar for large events, and so both are indicating similar behavior. Second, the difference in amplitude is modest when the event populations are the same, with an average of $~ 30 \%$. Splitting the difference would cover both datasets with a $\pm 15 \%$ uncertainty.

Surface slip-length measurements, in addition to sliplength estimates from magnitude-area, are not equivalent measures. Slip only begins to penetrate the surface for large events, with magnitudes $\mathbf{M}>6.5$ or so for strike-slip events, while average slip estimates from moment-area relations continue down to small events. It is only for the very largest events that we expect these two estimates to approach each other. However, because these are the events which dominate the sums, finding consistency between the two complementary views is important. Despite the remaining modest differences, the level of agreement we observe gives confidence to our understanding.

Inversions for slip distribution on modeled finite sources is one potential avenue for resolving the discrepancies noted here. Comparisons have been made using surface data and finite-source inversions (Mai and Beroza, 2000; Manighetti et al., 2007). One difficulty with this approach is the broad range of results obtained in the inversions from different groups for the same event. Given the vast differences, as well as uncertainties in the underdetermined inverse problem, we restrict our attention in this paper to the most constrained observations.

\section{Incorporating Results into Hazard}

As noted in the Introduction, magnitude-area scaling relations are used in two ways in standard seismic hazard estimates: to estimate the sizes of events, and to estimate the slip and therefore rates of the events. Given the independent but complementary and directly observable information provided by surface-slip measurements, we recommend expanding logic-tree branches in seismic hazard estimates to incorporate this information.

For maximal numbers of branches on a logic tree, all of the magnitude-area relations could be considered to estimate sizes; in addition, all of the magnitude-area and slip-length relations could be considered to estimate rates. Combining these two sets independently gives a number of branches equal to the product of the numbers of relations in the two sets independently. Table A4 (a) in Appendix A illus- trates this case, using the four magnitude-area scaling relations for sizes, and four slip-length relations for rates, consisting of two implied slip lengths from magnitude area and two slip lengths from surface slip. This gives a total of 16 branches.

Deciding which branches to use, or which to assign greater weight, depends on a number of factors. Goodness of fits to the data, number of parameters, and information theoretic measures of fits are all useful in guiding weights of logic-tree branches. Relative AIC takes into account both the gain in fit as well as the cost of additional parameters in fitting, and thus the fits of the scaling relations to the data are presented in the tables in Appendix A in rankordering of best AIC fit. Other considerations should go into weighting branches. We seek to span uncertainty in what is the best type of scaling (epistemic uncertainty), so a model which is different than the others deserves additional consideration.

The pruning of logic-tree branches can also be performed using additional criteria. One method is through consistency with both the magnitude-area and implied slip-length scaling. For example, the $S \sim L^{1 / 2}$ scaling in equation (19) matches the implicit scaling in the EB equation (10) magnitude-area relation. Table A4 (b) in Appendix A illustrates this case, which leads to a fairly substantial trimming of branches in Table A4 (a), leaving four. In the UCERF2 (WGCEP, 2008) case, this type of cut was made in that the same relation was used for both size and rate. That is, in the language of UCERF2, in the Earthquake Rate Models, illustrated in Figure 4 of the UCERF2 report (WGCEP, 2008), the mag-area relationships were also used in the $M_{0}$-rate balancing on the type A fault models. Thus, in UCERF2, since no surface-slip relations were used, only two total branches remained from the two magnitude-area relations considered. While the application of this work has focused on slip-rate estimates, which are more appropriate to well-characterized fault systems where slip rates on faults are sufficiently defined so that constraints can be applied, the consistency argument could also be used as a constraint for favoring various magnitude-area scaling relations to estimate the sizes of events. Thus, this work also has potential applicability beyond areas that have wellconstrained slip rates on faults.

While linear slip-length scaling was not favored by our results, continuity with previous estimates can also be considered. Table A4 (c) in Appendix A shows an expansion to include previously used branches in UCERF2 (WGCEP, 2008).

\section{Slip-Rate Budgeting}

A new approach to imposing integral constraints on matching fault-slip rates is proposed in this paper. We suggest using slip-rate rather than moment-rate balancing. This has the advantage of being able to incorporate new kinds of data, including data that is less sensitive to rupture-width uncertainties and is observable at the surface. 
Rate estimates depend on slip estimates. Unfortunately, slip estimates from surface-slip measurements and from magnitude-area derived estimates differ. However, there is a fairly simple way to parameterize the differences. In an attempt to reconcile surface slip with that of magnitude-area estimates, we observed no obvious magnitude dependence, which was an important factor. Furthermore, dynamic modeling detected no strong magnitude dependence for large events in the fraction of deep-moment occurrence (Shaw and Wesnousky, 2008). Both lines of evidence support a simple parameterization of an overall constant multiplicative factor-let us call it $\Lambda$-in an attempt to use either surface slip-length estimates or implied-slip estimates from the magnitude area in order to estimate rates. Generally, $\Lambda=1$ would be an uncorrected multiplicative factor, but $\Lambda>1$ could be used if it was believed that slip was underestimated by unconsolidated sediment or afterslip in surface creep, or $\Lambda<1$ if it was overestimated by deep-slip corrections. Importantly, the lack of substantial magnitude dependence allows for a simple single-parameter correction.

For complete slip-rate budgeting, we require not only the scaling of slip with size, but also the rates of events - the magnitude frequency distribution. Fortunately, we do not need to deal with this additional part of the problem in finding a best-fitting scaling relation. This is because the error metric we have used, the standard deviation of slip, is robust to the functional forms of slip considered and to the range of generally used magnitude-frequency distributions (Gutenberg-Richter $b=1$, or characteristic). Only if small events or creep were dominating the slip budget would we need to focus on fits in a regime other than the large-eventdominated regime we have examined.

In locations where surface creep is a substantial fraction of the slip budget, traditional magnitude-area estimates are more appropriate for slip-rate budgeting, and full weighting should be given to those branches of the logic tree. Fortunately, this is a rare situation for faults. Exceptions in California include the creeping sections of the San Andreas and Hayward faults, but these exceptions prove the rule as they are but a small fraction of the active faults considered in California hazard calculations (WGCEP, 2008).

\section{Depth Extent of Rupture: Relationship between $W$ and Seismogenic Depth}

Past working groups have used the seismogenic depth $H$ as a proxy for downdip width $W$ during large events. However, there is some uncertainty as to the precise relationship between $W$ and $H$. Dynamic ruptures are known to be able to push at least some distance into velocity-strengthening layers, so $W$ would be expected to some extent to be at least as large as $H$. Rolandone et al. (2004) found a deepening of seismicity following Landers. We suggest dealing with the uncertainty posed by the additional potential deepening with a constant proportionality parameter $\xi$ :

$$
W=\xi H / \sin \theta \text {. }
$$

In this expression, we also have explicitly written the geometric correction for the fault-dip angle $\theta$ to transform depth to downdip length. What values are good values to use for $\xi$ needs to be discussed. We can probably put one bound on $\xi$, since it is unlikely that $W$ would break less deep than $H$ :

$$
\xi \geq 1
$$

In principle, $\xi$ need not be a constant, and could be a function of $L$, rendering $W$ a function of $L$ (King and Wesnousky, 2007; Leonard, 2010). This further extension is worthy of additional study. It would, for example, allow the association of the HB magnitude-area scaling with a sublinear slip-length scaling; one could trade high implied slips instead for high implied downdip widths (although the widths associated with the longest ruptures might be considered problematic). It would, however, lead to additional moment-rate estimates for the same slip rates on faults.

\section{Conclusion}

We have presented a new way to use geologically observable surface-slip measurements in seismic hazard estimates. We have demonstrated how slip-length scaling complements magnitude-area scaling relations in the two different realms, where it is used in seismic hazard estimates. One method, estimating sizes of events, provides a consistency constraint in matching implied slip-length scaling of magnitude-area with surface slip-length observations, thus helping to distinguish candidate-scaling relations. A second method involves estimating rates of events. To help match imposed rate constraints on faults, we propose using slip-rate rather than moment-rate balancing as the integral constraint on faults. This use provides an alternative estimate, which avoids downdip width uncertainties and possible ambiguity of deep coseismic slip below the seismogenic layer being mapped onto shallower layers, and potentially biasing slip estimates from magnitude-area relations.

In examining the consistency of slip-length scaling implied by magnitude-area estimates with surface slip-length measurements, we found qualitative consistency in the functional forms. However, systematic quantitative differences with the magnitude-area estimates are, on average, $\sim 30 \%$ higher. This may be due to overestimating slip from magnitude area due to deep coseismic slip being mapped onto seismogenic layers, or by surface-slip measurements underestimating slip, perhaps due to poorly recorded slip in unconsolidated surface layers. Both of those biasing effects remain possibilities, and both are unresolved uncertainties. Nevertheless, the overall functional consistency lends support for the approach of using the complementary information contained in the slip-length data. 
Fitting slip-length scaling with different functional forms, we found evidence for sublinear slip scaling at the largest events and a breakdown of $L$ scaling, for both the implied slip from magnitude-area and the surface-slip data. In the surface-slip data, the best fitting was found for a constant stress-drop model. The next best fitting, which was nearly as good, was for a $L^{1 / 2}$ model. Fits were done using strike-slip data since reasonable populations of events were available to perform sufficient statistical fits. Nevertheless, what data there were for other focal mechanisms appeared to have behavior consistent with the strike-slip data.

In their seminal work, Wells and Coppersmith (1994) compared uncertainties in their regressions and determined that surface slip was much less reliable than other regressions. We also find large uncertainties when fitting with the power-law functional form implied by their scaling relations, but instead suggest this is a limitation of the functional forms they considered in trying to fit the data, not of the data itself. More constrained functional forms do appear to have much smaller parameter uncertainties and perform well in data parameterization. Moreover, the degree of misfit to surface-slip data is comparable to (and actually somewhat smaller) than the misfit to implied slip values derived from magnitude-area data. The relatively low scatter in the surface-slip data supports the value of these data.

A new scaling for slip versus length based on a constant stress-drop model was introduced. A generalization to allow for arbitrary rake and dip faults was presented. This constant stress-drop model provides a geometrical explanation for a longstanding puzzle of the large aspect ratio at which slip begins to saturate. The constant stress-drop model provided a very good fit to the slip-length data, finding support for a constant stress drop connecting an $L$-scaling to that of a $W$ scaling regime. The mean values of the stress drop were found to be consistent with that inferred from small events (Hanks, 1977; Rubin, 2002), providing support for the remarkable invariance displayed by earthquakes of constant stress-drop scaling from small to great earthquakes. The good fit of constant stress-drop scaling to the surface sliplength data was true not only of the mean, but the scatter around the mean. A remarkably small degree of scatter in the implied stress drops was found, with a COV $<0.5$ measured. This order factor of 2 difference from the mean in stress drops contrasts with a much larger variation, closer to a factor of 10 from the mean, observed seismologically in small events (Hanks, 1977). It may be that uncertainties in small-event measurements are dominating the scatter, with, in particular, cubic dependence on corner-frequency magnifying seismological estimates. Alternatively, it may be a real effect, and large and great earthquakes might have significantly reduced scatter in the intrinsic variability of the stress drops. Whatever the origin of the difference, the relatively small variability seen for large earthquake stress drops is beneficial for applications to seismic hazard estimates, where tighter constraints are more useful.

\section{Data and Resources}

All data are reproduced and available in the (E) electronic supplement to this article. Data source for WGCEP03 magnitude-area-width taken from published literature (Ellsworth, 2003; WGCEP, 2003), and reproduced in (E) Table S1. Data source for magnitude-area data from Hanks and Bakun $(2002,2008)$ is reproduced in (E) Table S2. Data source for slip-length data is adapted from Wesnousky (2008), with correction added for the 1857 M 7.8 Fort Tejon earthquake to reflect the recent LiDAR results of Zielke et al. (2010). We have also added the value for the 2008 M 7.9 Wenchuan earthquake $\mathrm{Xu}$ et al. (2009). These data are reproduced in (E) Table S3. A final (E) Table S4 contains a database derived from the sources used in $\mathrm{S} 2$ and $\mathrm{S} 3$ to construct a database of properties of the events common to these two databases. All four supplementary (E) Tables S1-S4 are available in the electronic supplement to this article.

\section{Acknowledgments}

The author would like to thank Ned Field in leading the UCERF effort which helped motivate this work. I thank a number of people for comments which helped improve the manuscript, particularly Martin Mai, Mark Stirling, Mark Leonard, Tom Hanks, and a couple of anonymous reviewers. This work was supported by the National Science Foundation's (NSF's) EAR-0911221, EAR-0934736, and EAR-0943939, by the Southern California Earthquake Center (SCEC), and by the California Earthquake Authority (CEA).

\section{References}

Akaike, H. (1974). A new look at the statistical model identification, IEEE Trans. Automatic Control, AC19, 716.

Allmann, B. P., and P. M. Shearer (2009). Global variations of stress drop for moderate to large earthquakes, J. Geophys. Res. 114, no. B01, 310, doi: $10.1029 / 2008 J B 005821$.

Bodin, P., and J. N. Brune (1996). On the scaling of slip with rupture length for shallow strike-slip earthquakes: Quasistatic models and dynamic rupture propagation, Bull. Seismol. Soc. Am. 86, 1292-1299.

Boggs, P. T., and J. E. Rogers (1990). Orthogonal distance regression, U.S. Dept. Commerce, NISTIR 89-4197.

Brune, J. N. (1970). Tectonic stress and the spectra of seismic shear waves from earthquakes, J. Geophys. Res. 75, 4997.

Das, S. (1982). Appropriate boundary-conditions for modeling very long earthquakes and physical consequences, Bull. Seismol. Soc. Am. 72, 1911.

Ellsworth, W. L. (2003). Magnitude and area data for strike-slip earthquakes, U.S. Geol. Surv. Open-File Rept. 03-214 Appendix D.

Graves, R., T. H. Jordan, S. Callaghan, E. Deelman, E. Field, G. Juve, C. Kesselman, P. Maechling, G. Mehta, K. Milner, D. Okaya, P. Small, and K. Vahi (2011). Cybershake: A physics-based seismic-hazard model for southern California, Pure Appl. Geophys. 168, 367, doi: 10.1007/s00024-010-0161-6.

Hanks, T. C. (1977). Earthquake stress-drops, ambient tectonic stresses, and the stresses that drive plates, Pure Appl. Geophys. 115, 441-458.

Hanks, T. C., and W. H. Bakun (2002). A bilinear source-scaling model for M-log A observations of continental earthquakes, Bull. Seismol. Soc. Am. 92, 1841.

Hanks, T. C., and W. H. Bakun (2008). M-log A observations of recent large earthquakes, Bull. Seismol. Soc. Am. 98, 490. 
Hillers, G., and S. G. Wesnousky (2008). Scaling relations of strike-slip earthquakes with different slip-rate dependent properties at depth, Bull. Seismol. Soc. Am. 98, 1085.

Hudnut, K. W., J. M. Fletcher, T. K. Rockwell, J. J. Gonzalez-Garcia, O. Teran, and S. O. Akciz (2010). Earthquake rupture complexity evidence from field observations (abstract T51E-02), Eos Trans. AGU 91, T51E-02.

King, G. L., and S. Wesnousky (2007). Scaling of fault parameters for continental strike-slip earthquakes, Bull. Seismol. Soc. Am. 97, 1833, doi: 10.1785/0120070048.

Knopoff, L. (1958). Energy release in earthquakes, Geophys. J. Roy. Astron. Soc. 1, 44.

Leonard, M. (2010). Earthquake fault scaling: Self-consistent relating of rupture length, width, average displacement, and moment release, Bull. Seismol. Soc. Am. 100, 1971, doi: 10.1785/0120090189.

Madariaga, R. (1976). Dynamics of an expanding circular crack, Bull. Seismol. Soc. Am. 66, 639

Mai, P. M., and G. C. Beroza (2000). Source scaling properties from finite rupture models, Bull. Seismol. Soc. Am. 90, 604-615.

Manighetti, I., M. Campillo, S. Bouley, and F. Cotton (2007). Earthquake scaling, fault segmentation, and structural maturity, Earth Planet. Sci. Lett. 253, 429.

Rolandone, F., R. Bürgmann, and R. M. Nadeau (2004). The evolution of the seismic-aseismic transition during the earthquake cycle: Constraints from the time-dependent depth distribution of aftershocks, Geophys. Res. Lett. 31, no. L23, 610, doi: 10.1029/2004GL021379.

Romanowicz, B. (1992). Strike-slip earthquakes on quasi-vertical transcurrent fault: Inferences for general scaling relations, Geophys. Res. Lett. 19, 481.

Romanowicz, B. (1994). Comment on 'A reappraisal of large earthquake scaling' by C. Scholz, Bull. Seismol. Soc. Am. 84, 1675.

Rubin, A. M. (2002). Aftershocks of microearthquakes as probes of the mechanics of rupture, J. Geophys. Res. 107, 2142, doi: 10.1029/ 2001JB000496.

Scholz, C. H. (1982). Scaling laws for large earthquakes: Consequences for physical models, Bull. Seismol. Soc. Am. 72, 1-14.

Scholz, C. H. (2002). The Mechanics of Earthquakes and Faulting, Second Ed., Cambridge Univ. Press, 202-207.

Shaw, B. E. (2009). Constant stress drop from small to great earthquakes in magnitude-area scaling, Bull. Seismol. Soc. Am. 99, 871, doi: 10.1785/ 0120080006.

Shaw, B. E. (2011). Surface-slip gradients of large earthquakes, Bull. Seismol. Soc. Am. 101, 792, doi: 10.1785/0120100053.

Shaw, B. E., and C. H. Scholz (2001). Slip-length scaling in large earthquakes: Observations and theory and implications for earthquake physics, Geophys. Res. Lett. 28, 2995.

Shaw, B. E., and S. G. Wesnousky (2008). Slip-length scaling in large earthquakes: The role of deep-penetrating slip below the seismogenic layer, Bull. Seismol. Soc. Am. 98, 1633.

Sieh, K. E. (1978). Slip along San Andreas fault associated with great 1857 earthquake, Bull. Seismol. Soc. Am. 68, 1421.

Starr, A. T. (1928). Slip in a crystal and rupture in a solid due to shear, Proc. Camb. Phil. Soc. 24, 489.

Wei, S., E. Fielding, S. Leprince, A. Sladen, J. P. Avouac, D. Helmberger, E. Hauksson, R. Chu, M. Simons, K. Hudnut, T. Herring, and R. Briggs (2011). Superficial simplicity of the $2010 M_{\mathrm{w}} 7.2$ El Mayor-Cucapah earthquake of Baja California, Mexico, Nature Geosci. 4, 615, doi: 10.1038/ngeo1213.

Wells, D. L., and K. J. Coppersmith (1994). New empirical relationships among magnitude, rupture length, rupture width, rupture area, and surface displacement, Bull. Seismol. Soc. Am. 84, 974.

Wesnousky, S. G. (2008). Displacement and geometrical characteristics of earthquake surface ruptures: Issues and implications for seismichazard analysis and the process of earthquake rupture, Bull. Seismol. Soc. Am. 98, 1609.

Working Group on California Earthquake Probabilities (WGCEP) (2003). Earthquake probabilities in the San Francisco Bay region: 20022031, U.S. Geol. Surv. Open-File Rept. 03-214.
Working Group on California Earthquake Probabilities (WGCEP) (2008). The Uniform California Earthquake Rupture Forecast, version 2 (UCERF2), U.S. Geol. Surv. Open-File Rept. 2007-1437.

Xu, X., X. Wen, G. Yu, G. Chen, Y. Klinger, J. Hubbard, and J. Shaw (2009). Coseismic reverse- and oblique-slip surface faulting generated by the $2008 M_{\mathrm{w}}$ 7.9 Wenchuan earthquake, China, Geology 37, 515, doi: 10.1130/G25462A.1.

Yen, Y. T., and K. F. Ma (2011). Source-scaling relationship for M 4.6-8.9 earthquakes, specifically for earthquakes in the collision zone of Taiwan, Bull. Seismol. Soc. Am. 101, 468, doi: 10.1785/ 0120100046

Zielke, O., J. R. Arrowsmith, L. G. Ludwig, and S. O. Akciz (2010). Slip in the 1857 and earlier large earthquakes along the Carrizo Plain, San Andreas fault, Science 327, 1119, doi: 10.1126/science.1182781.

\section{Appendix A}

\section{Fits of Scalings to Data and Logic-Tree Branches}

\section{Table A1: Magnitude-Area Scaling}

The standard deviation in Table A1 measures the difference in magnitudes of the data from the predicted curve. A least-squares fit is performed, which neglects errors in area relative to errors in magnitude. Uncertainties on parameters are shown for parameters fit to data; fixed parameters and parameters set in previous work or by other fits to data do not have associated uncertainties. Parameter uncertainties are estimated from ODR with large vertical values relative to horizontal data uncertainties. The number of free parameters is denoted by Number, followed by the best fitted parameter values. The parameter $C_{0}$ is the constant in the magnitudearea scaling relations. Ranking in this table, and those that follow, is by minimum AIC (Akaike, 1974), which rewards reduced standard deviation but penalizes additional parameters. AIC $=-2 \ln \mathcal{L}+2 k$, where $\mathcal{L}$ is the likelihood and $k$ is the number of fitted parameters. Thus, differences in AIC correspond to differences in log-likelihood, with relative likelihood of two models being $e^{-\Delta \mathrm{AIC} / 2}$. This gives a scale of the differences in AIC that matter, at least a few to be significant. In the table, we use AIC $=n\left[\ln \left(2 \pi \sigma^{2}\right)+1\right]+2 k$ where $\sigma$ is the standard deviation, $n$ is the number of data points, and $k$ is the number of parameters. For use in hazard estimates, scaling relations need to be able to operate in prospective mode, so fixing parameters, rather than refitting them with each new dataset, is preferred. Penalizing fits for the number of parameters when the parameters are not refit to the dataset does somewhat overestimate the AIC parameter penalty; however, given the small number of parameters in all the fits, the overestimate in AIC parameter penalty is not important at the levels being examined here. (a) Data from the WGCEP03 (Ellsworth, 2003; WGCEP, 2003). The modified WC scaling is denoted with a dagger symbol: $\mathrm{WC}^{\dagger}$. The S09 scaling with fixed values of $W$ is denoted with a prime symbol: $\mathrm{S}^{\prime}{ }^{\prime}$. (b) Only large magnitudes $M>6.5$ are considered, reducing the number of data points from 77 to 49 . Because the parameters are not refit to the reduced dataset, the parameter 
Table A1

Magnitude-Area Scaling Fits

\begin{tabular}{|c|c|c|c|c|c|c|c|}
\hline \multicolumn{2}{|c|}{ Scaling } & \multicolumn{2}{|l|}{ Misfits } & \multicolumn{4}{|c|}{ Parameters } \\
\hline Name & $\overline{\text { Equation }}$ & Standard Deviation & AIC & Number & & Values & \\
\hline \multicolumn{8}{|c|}{ (a) Fits to complete WGCEP03 magnitude-area-width dataset } \\
\hline S09 & (12) & 0.202 & -23.62 & 2 & $C_{0}=3.98$ & $\beta=7.4 \pm 2.3$ & \\
\hline $\mathrm{WC}^{\dagger}$ & (13) & 0.203 & -22.87 & 2 & $C_{0}=3.89 \pm 0.11$ & $C_{1}=1.08 \pm 0.04$ & \\
\hline S09' & (12) & 0.204 & -20.65 & 3 & $C_{0}=3.98$ & $\beta=7.4$ & $W=15 \mathrm{~km}$ \\
\hline $\mathrm{HB}$ & (11) & 0.212 & -16.13 & 2 & $C_{0}=3.98$ & $A_{c}=537 \mathrm{~km}^{2}$ & \\
\hline WC & (13) & 0.217 & -12.50 & 2 & $C_{0}=3.98$ & $C_{1}=1.02$ & \\
\hline EB & (10) & 0.229 & -6.15 & 1 & $C_{0}=4.20$ & & \\
\hline
\end{tabular}

(b) Misfits to $M>6.5$ subset of WGCEP03 data without refitting parameters

\begin{tabular}{|c|c|c|c|c|c|c|c|}
\hline EB & (10) & 0.173 & -31.09 & 1 & $C_{0}=4.20$ & & \\
\hline S09 & (12) & 0.177 & -26.23 & 2 & $C_{0}=3.98$ & $\beta=7.4$ & \\
\hline $\mathrm{WC}^{\dagger}$ & (13) & 0.178 & -26.32 & 2 & $C_{0}=3.89$ & $C_{1}=1.08$ & \\
\hline S09' & (12) & 0.190 & -17.60 & 3 & $C_{0}=3.98$ & $\beta=7.4$ & $W=15 \mathrm{~km}$ \\
\hline $\mathrm{HB}$ & (11) & 0.213 & -8.72 & 2 & $C_{0}=3.98$ & $A_{c}=537 \mathrm{~km}^{2}$ & \\
\hline WC & (13) & 0.216 & -7.17 & 2 & $C_{0}=3.98$ & $C_{1}=1.02$ & \\
\hline
\end{tabular}

(c) Fits to complete Hanks-Bakun magnitude-area dataset

$\begin{array}{llllllcc}\text { S09 }^{\prime} & (12) & 0.208 & -20.54 & 3 & C_{0}=3.98 & \beta=5.0 \pm 1.4 & W=19 \pm 3.4 \mathrm{~km} \\ \text { HB } & (11) & 0.213 & -18.46 & 2 & C_{0}=3.98 & A_{c}=537 \mathrm{~km}^{2} & \\ \text { WC }^{\dagger} & (13) & 0.215 & -16.43 & 2 & C_{0}=3.86 \pm 0.08 & C_{1}=1.08 \pm 0.03 & \\ \text { S09 }^{\prime} & (12) & 0.213 & -15.78 & 3 & C_{0}=3.98 & \beta=7.4 & W=15 \mathrm{~km} \\ \text { WC } & (13) & 0.220 & -12.32 & 2 & C_{0}=3.98 & C_{1}=1.02 & \\ \text { EB } & (10) & 0.275 & 24.23 & 1 & C_{0}=4.20 & \end{array}$

(d) Misfits to $M>6.5$ subset of Hanks-Bakun data without refitting parameters

\begin{tabular}{llllllll} 
EB & $(10)$ & 0.185 & -17.26 & 1 & $C_{0}=4.20$ & \\
WC $^{\dagger}$ & $(13)$ & 0.193 & -12.21 & 2 & $C_{0}=3.86$ & $C_{1}=1.08$ & \\
S09' $^{\prime}$ & $(12)$ & 0.200 & -7.58 & 3 & $C_{0}=3.98$ & $\beta=5.0$ & $W=19 \mathrm{~km}$ \\
S09' & $(12)$ & 0.204 & -6.32 & 3 & $C_{0}=3.98$ & $\beta=7.4$ & $W=15 \mathrm{~km}$ \\
HB & $(11)$ & 0.213 & -5.27 & 2 & $C_{0}=3.98$ & $A_{c}=537 \mathrm{~km}^{2}$ & \\
WC & $(13)$ & 0.216 & -4.05 & 2 & $C_{0}=3.98$ & $C_{1}=1.02$ & \\
\hline
\end{tabular}

penalties are perhaps overly harsh, since the parameters are not refit. However, the AIC values do offer some help as to the statistical significance of the differences in the standard deviations, so they are presented, nevertheless. This method favors to some degree the EB scaling, since it was designed to fit over this range, while the other scalings were fit over the whole range. (c) Data from the HB database (Hanks and Bakun, 2008). Because width information is unavailable, only S09'-fixed $W$ solutions are shown. Two of those are shown; one optimized for the HB data, and the other a default version based on the WGCEP03 data. (d) Only large magnitudes $M>$ 6.5 are considered, reducing the number of data points from 87 to 36 . Again, this favors the EB scaling.

\section{Table A2: Slip-Length Scaling from Implied Magnitude Area Data}

The standard deviation in Table A2 measures the difference in slip of the data from the predicted curve. The data are transformed from magnitude-area to slip-length as follows. To get slip, we convert magnitude to moment and divide by area and modulus to get slip. When length is available, we use it.
When it is not, we divide area by a default width, with width being the lesser of the length compared with downdip seismogenic width. To get length, we divide area by width, assuming width is the lesser of the length compared with the downdip seismogenic width. Note that we do not refit the parameters, which were set based on fits in log-space magnitude-log area, to the new linear space slip-length metric. All parameters refit to datasets shown with uncertainty estimates on parameters. (a) Transformed data from WGCEP03 data (WGCEP, 2003). Lengths from database. (b) Transformed data from HB database (Hanks and Bakun, 2008). Lengths from dividing area by default width, with seismogenic width of $15 \mathrm{~km}$. (c) Surface slip-length scaling relations are fit to the implied slip-length HB data. This is done for completeness, and comparison with the surface level in the different tables.

\section{Table A3: Slip-Length Scaling from Surface-Slip Data}

The standard deviation in Table A3 measures the difference in slip of the data from the predicted curve. The data is derived from the Wesnousky database for surface slip of 
Table A2

Implied Slip-Length from Magnitude-Area

\begin{tabular}{|c|c|c|c|c|c|c|}
\hline \multicolumn{2}{|c|}{ Scaling } & \multicolumn{2}{|l|}{ Misfits } & \multicolumn{3}{|c|}{ Parameters } \\
\hline Name & Equation & Standard Deviation & AIC & Number & Values & \\
\hline \multicolumn{7}{|c|}{ (a) Misfits without rescaling for new metric; from WGCEP03 data } \\
\hline S09 & (12) & 1.212 & 252.1 & 2 & $C_{0}=3.98$ & $\beta=7.4$ \\
\hline S09' & (12) & 1.245 & 256.3 & 2 & $C_{0}=3.98$ & $W \beta=15 \times 7.4=111 \mathrm{~km}$ \\
\hline EB & (10) & 1.275 & 258.0 & 1 & $C_{0}=4.20$ & \\
\hline $\mathrm{WC}^{\dagger}$ & (13) & 1.415 & 276.0 & 2 & $C_{0}=3.86$ & $C_{1}=1.08$ \\
\hline HB & (11) & 1.687 & 303.0 & 2 & $C_{0}=3.98$ & $A_{c}=537 \mathrm{~km}^{2}$ \\
\hline WC & (13) & 1.699 & 304.1 & 2 & $C_{0}=3.98$ & $C_{1}=1.02$ \\
\hline \multicolumn{7}{|c|}{ (b) Misfits without rescaling for new metric; from Hanks and Bakun (2008) data } \\
\hline S09' & (12) & 1.180 & 279.6 & 2 & $C_{0}=3.98$ & $W \beta=15 \times 7.4=111 \mathrm{~km}$ \\
\hline $\mathrm{EB}$ & (10) & 1.211 & 282.2 & 1 & $C_{0}=4.20$ & \\
\hline $\mathrm{WC}^{\dagger}$ & (13) & 1.282 & 294.1 & 2 & $C_{0}=3.86$ & $C_{1}=1.08$ \\
\hline $\mathrm{HB}$ & (11) & 1.459 & 316.6 & 2 & $C_{0}=3.98$ & $A_{c}=537 \mathrm{~km}^{2}$ \\
\hline WC & (13) & 1.549 & 327.0 & 2 & $C_{0}=3.98$ & $C_{1}=1.02$ \\
\hline \multicolumn{7}{|c|}{ (c) Surface slip scaling relations fit to Hanks-Bakun implied slip-length data } \\
\hline$L^{\eta}$ & (21) & 1.190 & 281.1 & 2 & $C_{8}=0.026 \pm 0.013 \mathrm{~km}^{1-2 \eta}$ & $\eta=0.632 \pm 0.061$ \\
\hline$L^{1 / 2}$ & (19) & 1.236 & 285.8 & 1 & $C_{6}=7.46 \pm 0.42 \cdot 10^{-5}$ & \\
\hline $\mathrm{S} 12$ & (22) & 1.249 & 287.6 & 1 & $\Delta \sigma=5.95 \pm 0.34 \mathrm{MPa}$ & \\
\hline$L$ & (20) & 1.339 & 299.7 & 1 & $C_{7}=1.19 \pm 0.08 \cdot 10^{-6} \mathrm{~km}^{-1}$ & \\
\hline
\end{tabular}

large strike-slip events (Wesnousky, 2008). (a) Shows a comparison of the surface slip data with scaling relations derived from transformed magnitude-area to implied sliplength. (b) Shows a comparison of the surface-slip data with scaling relations proposed for surface slip-length scaling. The best overall fit to the data, in terms of AIC, is constant stress-drop (S12) scaling. With respect to the power-law fits across the different datasets, note the parameter sensitivity of $\eta$ to the datasets, with $\eta$ varying substantially between fits to the surface-slip data here and the implied-slip data in Table A2. Note also the large ratio of the uncertainty in amplitude of the power-law fit relative to the amplitude itself. This is an undesirable quality of a measure and argues against this functional form. In contrast, the covariance of the fits $L^{1 / 2}, \mathrm{~S} 12$, and $L$ to the different datasets is substantial; they are stably varying together.

\section{Branches in Logic Tree}

Table A4 shows branches in logic trees. (a) Maximal branches showing four viable magnitude-area scaling relations combined with four viable slip-length scaling relations, for a total of 16 branches. (b) Trimmed set of branches using consistency in scaling assumptions, having basis of scaling in sizes being consistent with same assumptions underlying scaling in rates. This type of consistency was used in UCERF2. (c) Extension of trimmed consistency set to include previously used branch, which also provides role as outlier. Note: Default values unless otherwise specified are $W=15 \mathrm{~km}$ and $\mu=30 \mathrm{GPa}$. Tables are included in columns for examples of parameters one might use for scaling relations fit to datasets; other datasets would result in other values for scaling relation parameters.

\section{Appendix B}

\section{Parameters for Shaw09 Scaling Relation}

The Shaw09 (S09) parameters in equation (12) are set as follows. Because the S09 relation is meant to generalize the HB relation, we fix the constant parameter to equal the constant in the HB relation. This is not necessary, but uncertainties in the constant parameter are dominated by uncertainties in area estimates at small magnitudes, and no additional information in this regime has been added here; therefore, we adhere to continuity.

Substantial uncertainty exists concerning the relationship of seismogenic depth and rupture width. Because of these uncertainties, and because the S09 parameters use width information, we calibrate the parameters in a way that aims to best reproduce similar treatments of the inputs. The WGCEP03 database contains width information; therefore, we use that to calibrate the S09 $\beta$ parameter.

The width $W$ can be treated either as a fixed value and, thus, as a parameter in the S09 relation or as input from a given rupture. The advantage of using it as an input is that it allows for a broader range of applicability beyond the fixed value. It is also determined to do a better job fitting the data. Nevertheless, there are instances where one may want it to have a default value, such as during the examination of sensitivities and uncertainties in $W$ estimates, or when width information is unavailable, as it is in the HB database, which has only magnitude and area. 
Table A3

Slip Length from Surface-Slip Data

\begin{tabular}{|c|c|c|c|c|c|c|}
\hline \multicolumn{2}{|c|}{ Scaling } & \multicolumn{2}{|l|}{ Misfits } & \multicolumn{3}{|c|}{ Parameters } \\
\hline Name & Equation & Standard Deviation & $\overline{\text { AIC }}$ & Number & Values & \\
\hline \multicolumn{7}{|c|}{ (a) Implied slip-scaling relations } \\
\hline S09 & $(18)$ & 0.741 & 51.00 & 2 & $C_{5}=2.80 \pm 0.53 \times 10^{-5}$ & $H \beta=73.1 \pm 19.7 \mathrm{~km}$ \\
\hline EB & (15) & 0.804 & 52.44 & 1 & $C_{2}=4.91 \pm 0.38 \times 10^{-5}$ & \\
\hline HB & (17) & 1.171 & 68.22 & 1 & $C_{4}=1.65 \pm 0.20 \times 10^{-5}$ & \\
\hline \multicolumn{7}{|c|}{ (b) Surface-slip scaling relations } \\
\hline $\mathrm{S} 12$ & (22) & 0.760 & 50.05 & 1 & $\Delta \sigma=3.91 \pm 0.29 \mathrm{MPa}$ & \\
\hline$L^{1 / 2}$ & (19) & 0.804 & 52.43 & 1 & $C_{6}=4.91 \pm 0.38 \times 10^{-5}$ & \\
\hline$L^{\eta}$ & (21) & 0.788 & 53.60 & 2 & $C_{8}=0.093 \pm 0.069 \mathrm{~km}^{1-2 \eta}$ & $\eta=0.421 \pm 0.092$ \\
\hline$L$ & (20) & 1.174 & 68.34 & 1 & $C_{7}=7.10 \pm 0.88 \times 10^{-7} \mathrm{~km}^{-1}$ & \\
\hline
\end{tabular}

Table A4

Logic-Tree Branches

\begin{tabular}{|c|c|c|c|c|c|}
\hline \multicolumn{3}{|c|}{ Sizes } & \multicolumn{3}{|c|}{ Rates } \\
\hline Name & Equation & Table & Name & Equation & Table \\
\hline \multicolumn{6}{|c|}{ (a) Full branches } \\
\hline \multirow[t]{4}{*}{ S09 } & (12) & $1 \mathrm{a}$ & S09 & (18) & $2 a$ \\
\hline & & & EB & (15) & $2 a$ \\
\hline & & & $\mathrm{S} 12$ & (22) & $3 b$ \\
\hline & & & $L^{1 / 2}$ & (19) & $3 b$ \\
\hline \multirow[t]{4}{*}{ EB } & (10) & $1 \mathrm{a}$ & S09 & (18) & $2 \mathrm{a}$ \\
\hline & & & EB & (15) & $2 \mathrm{a}$ \\
\hline & & & S12 & (22) & $3 b$ \\
\hline & & & $L^{1 / 2}$ & (19) & $3 b$ \\
\hline \multirow[t]{4}{*}{ HB } & (11) & $1 \mathrm{a}$ & S09 & (18) & $2 \mathrm{a}$ \\
\hline & & & EB & (15) & $2 \mathrm{a}$ \\
\hline & & & S12 & (22) & $3 b$ \\
\hline & & & $L^{1 / 2}$ & (19) & $3 b$ \\
\hline \multirow[t]{4}{*}{$\mathrm{WC}^{\dagger}$} & (13) & 1a & S09 & (18) & $2 \mathrm{a}$ \\
\hline & & & EB & (15) & $2 \mathrm{a}$ \\
\hline & & & $\mathrm{S} 12$ & (22) & $3 b$ \\
\hline & & & $L^{1 / 2}$ & (19) & $3 b$ \\
\hline \multicolumn{6}{|c|}{ (b) Trimmed by consistency of scaling assumption } \\
\hline \multirow[t]{2}{*}{ S09 } & (12) & & S09 & (12) & \\
\hline & & & $\mathrm{S} 12$ & (22) & \\
\hline \multirow[t]{2}{*}{ EB } & (10) & & EB & (10) & \\
\hline & & & $L^{1 / 2}$ & (19) & \\
\hline \multicolumn{6}{|c|}{ (c) Extension of consistency set to previously used branch } \\
\hline \multirow[t]{2}{*}{ S09 } & (12) & $1 \mathrm{a}$ & S09 & (12) & 1a \\
\hline & & & $\mathrm{S} 12$ & (22) & $3 b$ \\
\hline \multirow[t]{2}{*}{ EB } & (10) & $1 \mathrm{a}$ & EB & (10) & $1 \mathrm{a}$ \\
\hline & & & $L^{1 / 2}$ & (19) & $3 b$ \\
\hline HB & (11) & 1a & $\mathrm{HB}$ & (11) & $1 \mathrm{a}$ \\
\hline
\end{tabular}

To find a default $\beta$ value, we fix $W$, since the pre-event area based on seismogenic depth is more akin to a default $W$ value. California fault values have an average $W \sim 11 \mathrm{~km}$, so we take that as the fixed value. To avoid mixing in substantially bigger $W$ 's, we restrict the WGCEP03 (WGCEP, 2003) dataset on which we fit $\beta$ to $W<15 \mathrm{~km}$. For these events with widths comparable to California events, we obtain for $W=11 \mathrm{~km}$ a best fit $\beta=7.4$ using least-squares magnitude misfits. There is quite a broad minimum, and other values could also be used with little degradation in misfit. This does, however, give a means of fixing a value for $\beta$, and if it performs well on other datasets, it will be a satisfactory parameter choice. ODR fits using all the WGCEP03 dataset give $\beta=7.7 \pm 2.0$, which is an alternative means of fixing $\beta$, well within the broad range of uncertainties in $\beta$.

For the HB dataset, because only area is provided, a fixed value of $W$ is used. We use a default value of $W=15 \mathrm{~km}$ for this global dataset, which includes some ruptures believed to be much wider than typical values for California faults. A good job fitting the data would indicate this is a reasonable choice.

Lamont Doherty Earth Observatory

Columbia University

Palisades, New York 10964 